\title{
Antifungal Potency and Modes of Action of a Novel Olive Tree Defensin Against Closely Related Ascomycete Fungal Pathogens
}

\author{
Hui Li, Siva L. S. Velivelli, and Dilip M. Shah ${ }^{+}$ \\ Donald Danforth Plant Science Center, Saint Louis, MO 63132, U.S.A.
}

Accepted 16 August 2019.

\begin{abstract}
Antimicrobial peptides play a pivotal role in the innate immunity of plants. Defensins are cysteine-rich antifungal peptides with multiple modes of action. A novel Oleaceae-specific defensin gene family has been discovered in the genome sequences of wild and cultivated species of a perennial olive tree, Olea europaea. OefDef1.1, a member of this defensin family, potently inhibits the in-vitro growth of ascomycete fungal pathogens Botrytis cinerea and three Fusarium spp. OefDef1.1 rapidly permeabilizes the plasma membrane of the conidial and germling cells of $\boldsymbol{B}$. cinerea. Interestingly, it induces reactive oxygen species and translocates to the cytoplasm only in the germlings but not in the conidia. In medium containing a high concentration of $\mathrm{Na}^{1+}$, antifungal activity of OefDef1.1 is significantly reduced. Surprisingly, a chimeric OefDef1.1 peptide containing the $\gamma$-core motif of a Medicago truncatula defensin, MtDef4, displays $\mathrm{Na}^{1+}$-tolerant antifungal activity. In a phospholipid-protein overlay assay, the chimeric peptide exhibits stronger binding to its phosphoinositide partners than OefDef1.1 and is also more potent in inhibiting gray mold disease on the surface of Nicotiana benthamiana and lettuce leaves than OefDef1.1. Significant differences are observed among the four ascomycete pathogens in their responses to OefDef1.1 in growth medium with or without the elevated concentration of $\mathrm{Na}^{1+}$. The varied responses of closely related ascomycete pathogens to this defensin have implications for engineering disease resistance in plants.
\end{abstract}

Keywords: antifungal, defensin, modes of action, olive tree, fungal pathogens

Antimicrobial peptides (AMPs) are recognized as important mediators of innate immunity in the plant kingdom, providing a first line of defense against fungal and oomycete pathogens (Goyal and Mattoo 2014; van der Weerden et al. 2013). Among several classes of AMPs expressed in plants, small cysteinerich defensins have been extensively studied for their

${ }^{\dagger}$ Corresponding author: D. M. Shah; dshah@danforthcenter.org

Funding: We are deeply grateful to Intrexon Company for supporting this research.

*The $\boldsymbol{e}$-Xtra logo stands for "electronic extra" and indicates that 12 supplementary figures and three supplementary tables are published online.

The author(s) declare no conflict of interest.

๑) 2019 The American Phytopathological Society antimicrobial properties, modes of action (MOA), and ability to provide protection from fungal and oomycete pathogens in crops (Cools et al. 2017; De Coninck et al. 2013; Kaur et al. 2011; Lacerda et al. 2014; Parisi et al. 2019). There are now approximately 1,200 plant defensin sequences in the Defensins Sequence Space database (Shafee et al. 2016). A vast majority of these peptides remain to be studied at a functional level. Plant defensins are 45 to 54 amino acids in length and contain an invariant tetradisulfide array that confers stability to their pseudo-cyclic backbone. Because of their conserved cysteine signature, plant defensins share a similar three-dimensional (3D) structure comprising a triple-stranded $\beta$-sheet and one $\alpha$-helix, suggesting they evolved from a common ancestor (van der Weerden and Anderson 2013). Despite their structural similarity, plant defensins are highly varied in their primary amino acid sequences.

The best-known property of cationic plant defensins is their ability to inhibit the growth of fungal pathogens in vitro and in planta. However, the MOA of only a small number of plant defensins have been studied in detail (Cools et al. 2017; Parisi et al. 2019). It is now recognized that plant defensins act using different MOA but, ultimately, cause membrane disruption. Some plant defensins act extracellularly on fungi and bind to specific cell wall or plasma membrane resident sphingolipids, disrupt membrane integrity, and activate cellular toxicity pathways. For example, the radish defensin RsAFP2 binds to glucosylceramide and this interaction results in the induction of cell-wall stress, accumulation of ceramides and reactive oxygen species (ROS), and, ultimately, cell death (Thevissen et al. 2012). Recently, several plant defensins that gain entry into fungal cells and target plasma membrane resident phospholipids have been characterized (Islam et al. 2017b; Parisi et al. 2019). Plant defensins such as NaD1 from Nicotiana alata and MtDef5 from Medicago truncatula recruit one or more phospholipids to oligomerize, induce membrane disruption, bind to intracellular targets, and trigger fungal cell death (Cools et al. 2017; Islam et al. 2017b; Poon et al. 2014).

The important question related to the antifungal action of plant defensins is whether their fungicidal mechanisms are conserved in different fungi. Previously, we reported evidence that mechanisms used by MtDef4 from M. truncatula to inhibit the growth of Fusarium graminearum and Neurospora crassa are not the same, even though these two fungi both belong to phylum Ascomycota, subphylum Pezizomycotina, and order Sordariomycetes (El-Mounadi et al. 2016). This raises the possibility that the architecture and composition of the cell wall and plasma membrane may be different, even in closely related fungal pathogens, and markedly influence the antifungal activity of plant defensins. But it is still unknown if different 
developmental stages of a fungal pathogen also respond differently to a plant defensin.

To date, plant defensins that have been studied in-depth for their MOA are mostly from short-lived herbaceous annual plants (Cools et al. 2017; Parisi et al. 2019). To our knowledge, very few defensins from perennial woody plants have been studied for their antifungal activity and MOA. The MOA of two members of the grapevine (Vitis vinifera) defensin gene family have been partially investigated (Giacomelli et al. 2012; Nanni et al. 2014). Olive tree (Olea europaea) is an evergreen woody perennial oil crop that is cultivated in Mediterranean countries for its healthy oil. The genome sequences of two cultivated and one wild ancestral olive tree (Barghini et al. 2014; Cruz et al. 2016; Unver et al. 2017) as well as the European ash tree (Fraxinus excelsior) (Sollars et al. 2017), all belonging to the family Oleaceae, have been sequenced and annotated. In this study, we have searched the genome sequences of the wild olive tree (O. europaea var. sylvestris) (Unver et al. 2017), a cultivated olive tree (O. europaea 'Farga') (Cruz et al. 2016), and F. excelsior (Sollars et al. 2017) and have identified several genes encoding defensins. The genomes of these trees contain a unique gene family encoding defensins not present in other eudicots.

In this study, we examined the structure-activity relationships and MOA of the antifungal defensin OefDef1.1, a member of the $O$. europaea 'Farga' defensin family. We show that the conidia and germlings of a necrotrophic fungal pathogen, Botrytis cinerea, respond differently to the antifungal action of this defensin. Based on our results, a model for the antifungal action of OefDef1.1 against conidia and germlings of this pathogen is proposed. A chimeric OefDef1.1 containing the $\gamma$-core motif of plant defensin MtDef4 exhibits cation-resistant antifungal activity in vitro and confers better resistance to $B$. cinerea than the wildtype OefDef1.1. We also report a comparative analysis of the antifungal properties of this defensin against a panel of closely related ascomycete fungal pathogens in the absence and presence of elevated levels of $\mathrm{Na}^{1+}$.

\section{RESULTS}

\section{Identification of a novel defensin gene family in olive and European ash trees.}

We set out to systematically identify defensin genes in the recently published annotated genome sequences of a cultivated olive tree ( $O$. europaea 'Farga'), a wild olive tree called oleaster (O. europaea var. sylvestris) (Cruz et al. 2016; Unver et al. 2017), and the European ash tree (F. excelsior), members of the family Oleaceae, using the BLAST search described below. Several members of the defensin family in the olive and European ash trees were identified and phylogenetic relationships among them were determined (Fig. 1). The $O$. europaea 'Farga' genome encodes eight defensins, and the $O$. europaea var. sylvestris genome encodes 15 defensins grouped into three clades. Phylogenetic analysis revealed that eight defensins (clade I) are homologs of MtDef4 from Medicago truncatula (Sagaram et al. 2011), six defensins (clade II) are homologs of MtDef5 from M. truncatula (Islam et al. 2017b), and nine defensins rich in histidine and tyrosine are clustered in a separate clade III on the phylogenetic tree (Fig. 1). The clade I defensins have comparable homologs in all higher plants (Sagaram et al. 2011), whereas clade II has comparable homologs in several other dicotyledonous plants (Islam et al. 2017a and b). Of the nine defensins present in clade III, only two (OE3B065497T1 and OE3B086095T1) are found in O. europaea 'Farga', while the remaining seven are found in O. europaea var. sylvestris. Thus, the cultivated olive tree with a smaller genome also has a smaller number of the clade III defensins. Four clade III defensins were also identified in the genome sequence of the European ash tree. These defensins share 83 to $88 \%$ sequence identity with OefDef1.1 defensin from $O$. europea 'Farga'. All clade III defensins have a high predicted net charge of 7.6 to 10.3 at $\mathrm{pH} 7.0$ and four predicted disulfide bonds with a conserved cysteine-stabilized $\alpha \beta$ signature. They share more than $80 \%$ sequence identity and contain a noncanonical cationic $\gamma$-core motif that contains 10 residues $\left(\mathrm{GXCX}_{10} \mathrm{C}\right)$, instead of the expected three to nine, between the two cysteine residues. Based on our analysis, we propose that clade III defensins represent a novel Oleaceaespecific gene family.

\section{OefDef1.1 exhibits potent in vitro and semi-in planta antifungal activity.}

The amino acid sequence of OefDef1.1 (OE3B065497T1) harboring a net charge of 9.3 is shown in Supplementary Fig. S1. In-vitro antifungal activity of this peptide against $B$. cinerea and three Fusarium spp. was determined. It inhibited the growth of these fungi with the half-maximal inhibitory concentration $\left(\mathrm{IC}_{50}\right)$ values of $1.6 \pm 0.6 \mu \mathrm{M}$ for $F$. graminearum, $1.1 \pm 0.2 \mu \mathrm{M}$ for F. virguliforme, $0.4 \pm 0.1 \mu \mathrm{M}$ for $F$. oxysporum, and $0.7 \pm 0.3 \mu \mathrm{M}$ for $B$. cinerea (Table 1). Growth inhibition was also observed under the microscope, which showed that treatment of $B$. cinerea conidia with $1.5 \mu \mathrm{M}$ OefDef1.1 completely inhibited their germination (Fig. 2A). In addition, a resazurin cell-viability assay was used to determine the minimal inhibitory concentration (MIC) at which concentration OefDef1.1 caused $100 \%$ cell death. Results indicated that cell death was observed at a concentration of $1.5 \mu \mathrm{M}$ OefDef1.1 in B. cinerea (Fig. 2B). Thus, OefDef1.1 has potent broad-spectrum antifungal activity.

Since OefDef1.1 inhibits the growth of $B$. cinerea in vitro, we evaluated its ability to reduce the symptoms of the gray mold disease semi-in planta. Different concentrations of the peptide were applied on the surface of the detached lettuce leaves followed immediately with the inoculum of fresh $B$. cinerea conidia. After $48 \mathrm{~h}$, leaves treated with no peptide showed large lesions; however, higher concentrations of peptides produced significantly smaller lesions of fungal infection (Fig. 2C and D). OefDef1.1 at $6 \mu \mathrm{M}$ almost completely prevented development of disease lesions. The relative DNA content of $B$. cinerea was also determined by quantitative (q)PCR. This analysis confirmed a significant decrease in the biomass of $B$. cinerea at a peptide concentration of $1.5 \mu \mathrm{M}$, although a more pronounced decrease in the biomass was observed at a peptide concentration over $6 \mu \mathrm{M}$ (Fig. 2E). Thus, surface application of OefDef1.1 efficiently reduces gray mold symptoms on lettuce leaves.

\section{OefDef1.1 rapidly permeabilizes the plasma membrane of the conidia and germlings of $B$. cinerea.}

Since $B$. cinerea is an economically important pathogen responsible for causing extensive pre- and postharvest decay in fruits, vegetables, and flowers (Dean et al. 2012), we have chosen it as a model fungus to elucidate the MOA of OefDef1.1.

Killing of fungal cells by antifungal plant defensins often correlates with the permeabilization of the plasma membrane. A SYTOX Green (SG) uptake assay was used to determine the effect of OefDef1.1 on plasma membrane integrity in real-time in the cells of $B$. cinerea. Fresh conidia and germlings were incubated with $3 \mu \mathrm{M}$ OefDef1.1, and SG uptake was monitored continuously by time-lapse confocal microscopy. In fresh conidia, the SG uptake was apparent within 4 min and increased with time (Fig. 3). After $20 \mathrm{~min}$, the nuclei were completely stained with SG, indicating rapid permeabilization of the conidial cells caused by OefDef1.1. OefDef1.1 also permeabilized the plasma membrane of germlings rapidly. SG signal was observed in germlings as early as 1 min following the peptide challenge (Fig. 3). No SG signal was observed in 
the conidia or germlings treated with water alone (Supplementary Fig. S2).

The conidia and germlings of $B$. cinerea differ in the subcellular localization of OefDef1.1 and ROS production.

We sought to determine if OefDef 1.1 acts extracellarly or if it enters the cytoplasm in the conidia and germlings of $B$. cinerea.
OefDef1.1 labeled with the fluorophore DyLight 550 was added to freshly harvested conidia. As shown in Figure 4A, OefDef1.1 bound to the cell wall within 10 min of challenge, and it remained bound to the cell wall even after $130 \mathrm{~min}$ of challenge. If labeled peptide was added to conidia along with the membrane-selective dye FM4-64, the DyLight 550 signal did not colocalize with the FM4-64 signal (Supplementary Fig.

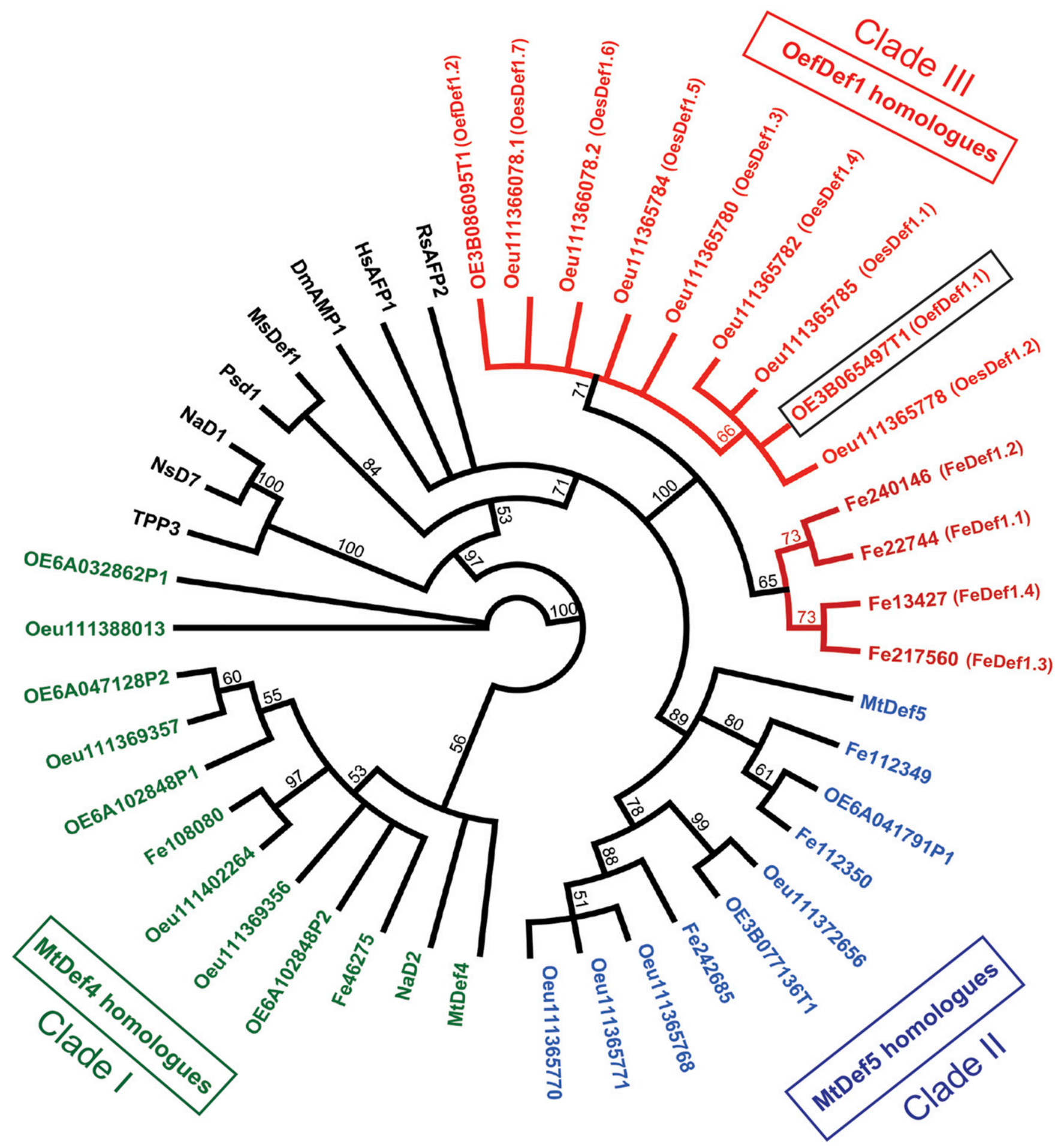

Fig. 1. Phylogenetic tree of defensin sequences identified from olive tree. The olive tree defensin homologs were identified through BLAST on Phytozome and National Center for Biotechnology Information. Geneious software was used to generate the phylogenetic tree of defensins based on the neighbor-joining method with bootstrap (10,000 replications). Defensin sequences are clustered into different clades: MtDef4 homologs (Clade I), MtDef5 homologs (Clade II), and OefDef1.1 homologs (Clade III). Among the clade III group, sequences of OefDef1.1 and OefDef1.2 are from Olea europaea 'Farga', FeDef1.1, FeDef1.2, FeDef1.3, and FeDef1.4 are from Fraxinus excelsior, and the other sequences are from wild olive tree O. europaea var. sylvestris. Clade I and II defensins have corresponding homologs in other plants. 
S3). No DyLight 550-labeled OefDef1.1 signal was detected in the cytoplasm of the conidia, indicating that, in freshly harvested conidia, OefDef1.1 acts via the extracellular side.

As observed in conidia, DyLight 550-labeled OefDef1.1 bound to the cell wall of $B$. cinerea germlings within $10 \mathrm{~min}$ after challenge (Fig. 4A). However, after $40 \mathrm{~min}$ of challenge, the color of cytoplasm became dark and germlings shrunk suddenly. The distance between cell walls decreased from 6.3 to $5.6 \mathrm{~nm}$, accompanied by shrinkage in the cell volume (Fig. 4A and B). After 40 min, DyLight 550-labeled OefDef1.1 began to enter into the cytoplasm of germling cells and, at $130 \mathrm{~min}$, filled the cells uniformly (Fig. 4A).

The induction of oxidative stress by antifungal peptides contributes to cell death in fungi. We determined whether OefDef1.1 induces ROS production in the conidia and germlings of $B$. cinerea. In conidia and germlings, ROS signal was observed following the OefDef1.1 treatment for $90 \mathrm{~min}$. However, a significant difference in ROS levels was observed between the two cell types (Fig. 4C and D). The ROS signal did not accumulate significantly in fresh conidia upon OefDef1.1 challenge but increased ninefold in germlings (Fig. 4D). These results indicate that germlings are more sensitive to OefDef1.1 than conidia and that ROS likely play a role in killing of germlings.

\section{Homology-based 3D structure of OefDef1.1 and analysis of its $\boldsymbol{\gamma}$-core motif variants.}

Using the solution structure of Artemisia vulgaris Artv1 defensin (Protein Data Bank [PDB] 2KPY), a homology model of the 3D structure of OefDef1.1 was generated and refined (Fig. 5A; Supplementary Fig. S4). The high-quality predicted structure was used for further analysis. As expected, the predicted structure of OefDef1.1 contains the structural characteristics found in other plant defensins for which structural information exists.

As reported, the highly conserved $\gamma$-core motif plays an important role in antifungal activity, phospholipid binding, and oligomer formation of a plant defensin. To determine to what extent the $\gamma$-core motif contributes to antifungal activity of OefDef1.1, we first generated two variants, OefDef1.1_V1 and OefDef1.1_V2, in which this motif was replaced by the corresponding $\gamma$-core motif of MtDef4 from $M$. truncatula and that of DmAMP1 from Dahlia merckii (Thevissen et al. 2003), respectively (Fig. 5B; Supplementary Table S1). The $\gamma$-core motif of MtDef4 (GRCRGFRRRC) is very different from that of OefDef1.1 in sequence and is more cationic. DmAMP1 sequence is $41 \%$ similar to the OefDef 1.1 sequence, but its $\gamma$-core motif (GACHVRNGKHMC) is also different in sequence from that of OefDef1.1. Antifungal activity of the variants against $B$. cinerea and $F$. oxysporum was determined (Fig. 5C). OefDef1.1_V1, which contains the $\gamma$-core motif of MtDef4, lost about $50 \%$ of activity compared with the wild-type OefDef 1.1 . It inhibited the growth of these fungi with an $\mathrm{IC}_{50}$ value of 2 to $3 \mu \mathrm{M}$, indicating an approximately threefold decrease in

Table 1. Half-maximal inhibitory concentration $\left(\mathrm{IC}_{50}\right)$ and minimal inhibitory concentration (MIC) values of OefDef1.1 against fungal pathogens $^{\mathrm{a}}$

\begin{tabular}{lcc}
\hline Pathogens & IC $_{\mathbf{5 0}}(\boldsymbol{\mu M})$ & MIC $(\boldsymbol{\mu M})$ \\
\hline F. graminearum & $1.6 \pm 0.6$ & 3 \\
F. virguliforme & $1.1 \pm 0.2$ & 3 \\
F. oxysporum & $0.4 \pm 0.1$ & 1.5 \\
B. cinerea & $0.7 \pm 0.3$ & 1.5 \\
\hline
\end{tabular}

${ }^{\mathrm{a}} \mathrm{IC}_{50}$ values were calculated from the inhibition curve for each fungal pathogen Values are mean \pm standard deviation; $n=3$. MIC values were determined by the resazurin cell-viability assay.
$B$. cinerea and a sevenfold decrease in $F$. oxysporum compared with wild-type OefDef1.1. In contrast, OefDef1.1_V2 containing the $\gamma$-core motif of DmAMP1 showed slightly higher antifungal activity than the wild-type OefDef1.1.

We also performed alanine scanning mutagenesis of the sequence LKNRHSKHYG between the two cysteines in the $\gamma$-core motif of OefDef1.1. Thus, three variants, designated OefDef1.1_V3 (OefDef1.1 LKNRHSKHYG/AAAHSKHYG), OefDef1.1 V4 (OefDef1.1 ${ }^{\text {LKNRHSKHYG/LKNAAAKHYG), and OefDef1.1_V5 }}$ (OefDef1.1 $1^{\text {LKNRHSKHYG/LKNRHSAAAA) }}$, were generated as shown in Supplementary Figure S5. These variants were expressed in Pichia pastoris and were purified using the same methods as for the wild-type OefDef1.1. Mass spectrometry confirmed the actual mass of each variant to be very close to the predicted mass (Supplementary Fig. S6). The purity of each variant was also confirmed using sodium dodecyl sulphatepolyacrylamide gel electrophoresis (Supplementary Fig. S7). Surprisingly, none of these variants lost in vitro antifungal activity. In fact, OefDef1.1_V3 and OefDef1.1_V4, displayed antifungal activity similar to that of the wild-type OefDef1.1, and OefDef1.1_V5 was even more potent than the wild-type OefDef1.1. These results suggest that specific amino acid residues within the $\gamma$-core motif are not critical for the antifungal activity of OefDef1.1 and there is some flexibility in the sequence requirement of this peptide. In addition, hydrophobicity rather than the net charge may be a more significant contributor to the antifungal activity of this peptide.

\section{OefDef1.1_V1 is more effective than OefDef1.1 in reducing gray mold disease symptoms when applied on Nicotiana benthamiana and lettuce leaves.}

Earlier, we showed that wild-type OefDef1.1, when applied topically on the detached leaves of iceberg lettuce, significantly inhibited gray mold disease symptoms (Fig. 2C, D, and E). We decided to compare the semi-in planta antifungal activity of OefDef1.1_V1, OefDef1.1_V2, and OefDef1.1_V5 with that of the wild-type OefDef1.1. Because of their large size and susceptibility to $B$. cinerea, detached $N$. benthamiana leaves were used in this assay to compare the antifungal activity of all peptides on the same leaf. The wild-type OefDef 1.1 and variants at different concentrations were applied to the surface of the leaf and it was infected immediately with the conidia of B. cinerea. Both lesion size and photosynthetic efficiency were measured. To avoid leaf-to-leaf variation, all four peptides along with a no-peptide control were spotted on the surface of the same leaf. Based on the Fv/Fm value, the condition of each infected leaf was rated as healthy $(\mathrm{Fv} / \mathrm{Fm}>0.7)$, slightly damaged (Fv/Fm 0.5 to 0.7$)$, or severely damaged $(\mathrm{Fv} / \mathrm{Fm}<0.5)$ (Supplementary Fig. S8). Compared with the no-peptide control, each peptide reduced the size of lesions caused by infection of the pathogen (Fig. 6A and B). As expected, the percentage of damaged area resulting from gray mold disease on each leaf decreased with increasing concentrations of each peptide (Fig. 6C). Among the four tested peptides, OefDef1.1_V2 showed similar efficacy in reducing disease lesions to the wild-type OefDef1.1, although the Fv/Fm data suggested that OefDef1.1_V2 was more potent at lower concentrations (3 and $6 \mu \mathrm{M})$. Surprisingly, OefDef1.1_V1, with much reduced antifungal activity in vitro, was most effective in inhibiting gray mold disease symptoms. At $3 \mu \mathrm{M}$ OefDef1.1_V1, an $80 \%$ reduction in lesion size was observed, and, at a concentration of $6 \mu \mathrm{M}$, disease symptoms were completely attenuated (Fig. 6A). OefDef1.1_V5, which exhibited more potent in vitro antifungal activity than wild-type OefDef1.1, was effective in reducing disease symptoms only at a high concentration of $24 \mu \mathrm{M}$ semiin planta. Similar results were also observed in lettuce (Fig. 6D and E). Thus, OefDef1.1_V1 with the higher net charge is more 
A
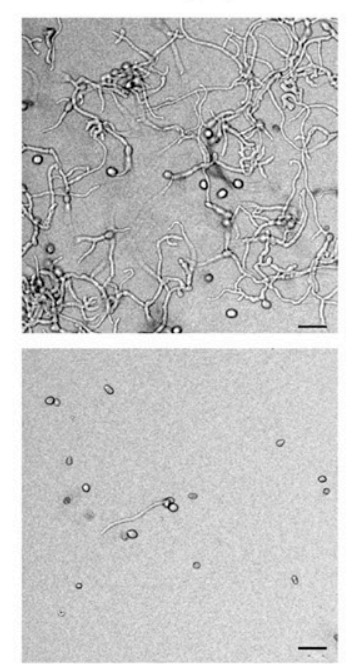

$1.5 \mu \mathrm{M}$
$0.375 \mu \mathrm{M}$
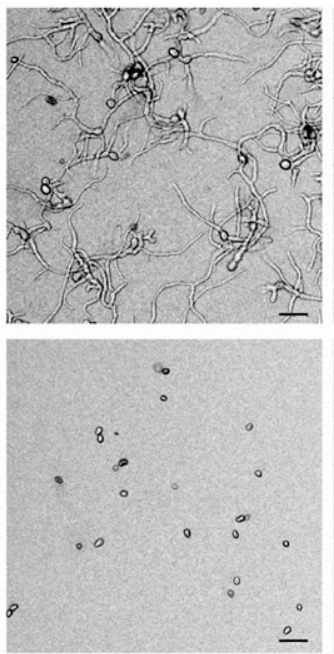

$3 \mu \mathrm{M}$
$0.75 \mu \mathrm{M}$
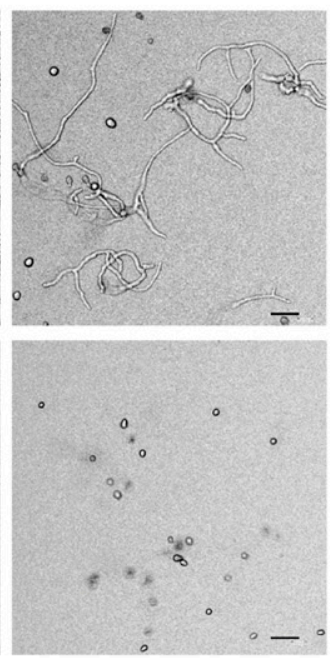

$6 \mu \mathrm{M}$
B

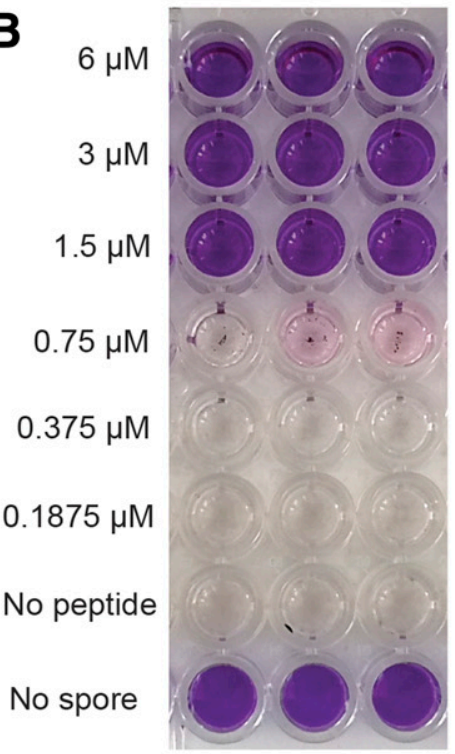

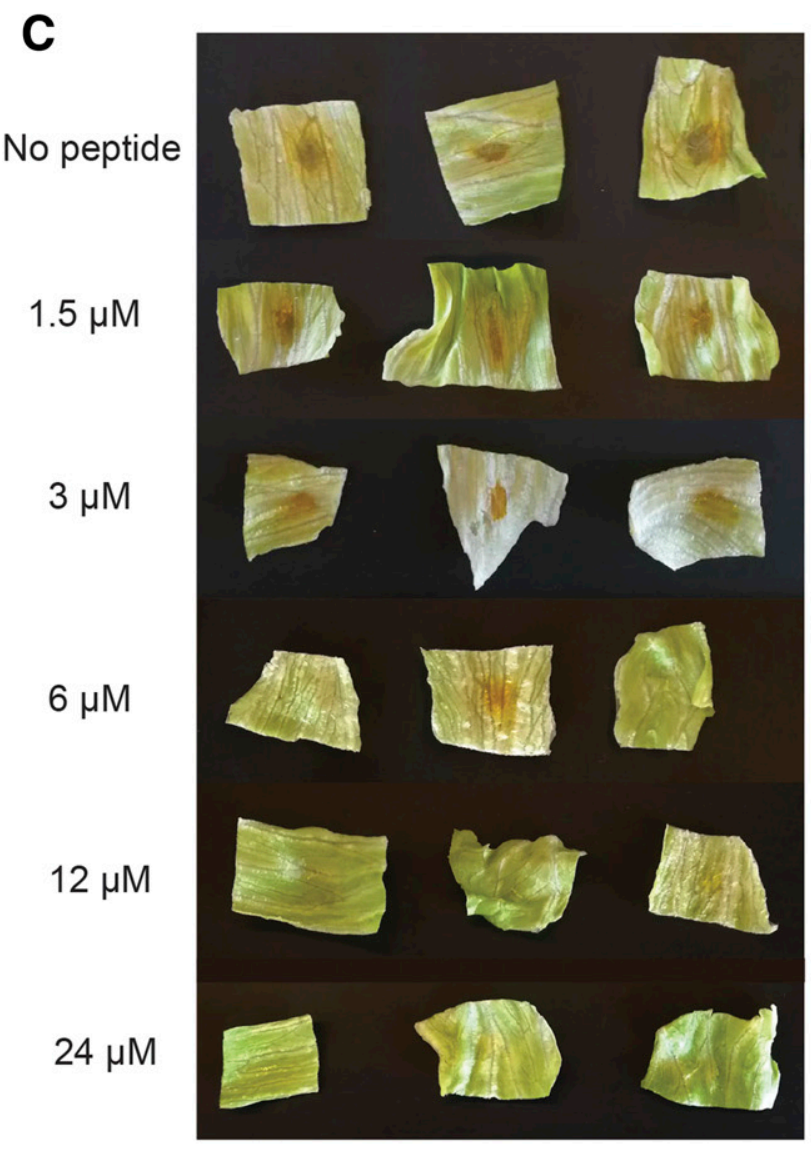
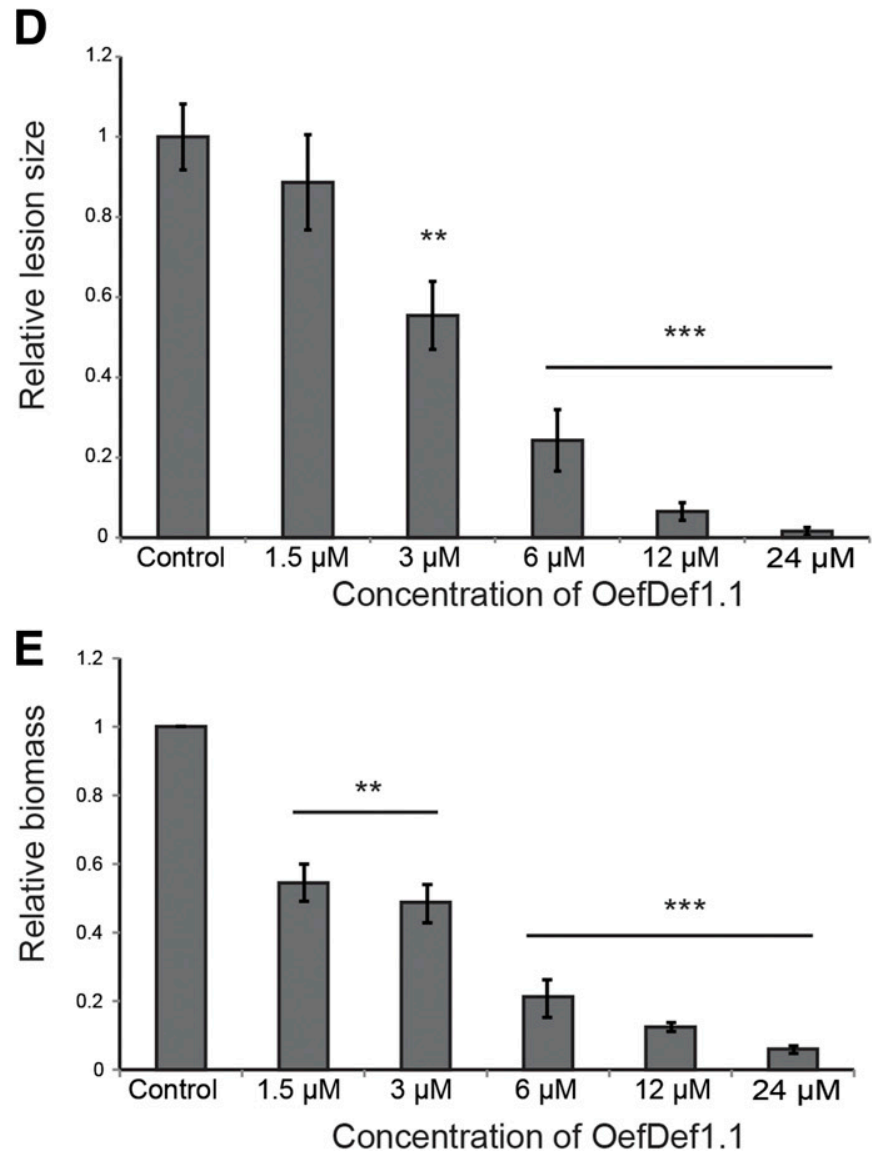

Fig. 2. OefDef1.1 exhibits broad-spectrum antifungal activity in vitro and reduces gray mold disease symptoms semi-in planta. A, Micrographs displaying growth inhibition of Botrytis cinerea at different concentrations of OefDef1.1 are shown. Complete inhibition of spore germination was observed at a concentration of $1.5 \mu \mathrm{M}$. Bar $=40 \mu \mathrm{m}$. Images were taken at $60 \mathrm{~h}$ postinoculation (hpi). B, Fungal cell death in $B$. cinerea was assessed using the resazurin cellviability assay. The wells with dark color indicated absence of live cells. $\mathbf{C}$, Lesions caused by $B$. cinerea infection of the detached lettuce leaves were progressively reduced in size with increasing concentrations of OefDef1.1. Leaves with no peptide applied were used as controls. Images were taken at 48 hpi. D, Relative lesion sizes at different concentrations of OefDef1.1 were measured, using ImageJ software. E, Relative fungal biomass indicated by the relative fungal DNA content was determined by quantitative PCR. Leaves without peptide treatment were used as controls. The statistical analysis was performed using two-tailed unpaired $t$ test to determine whether the observed differences were statistically significant (two asterisks $[* *]$ indicate $P<0.01$, three $[* * *$ ], $P<$ 0.001). Error bars denote standard deviations. 


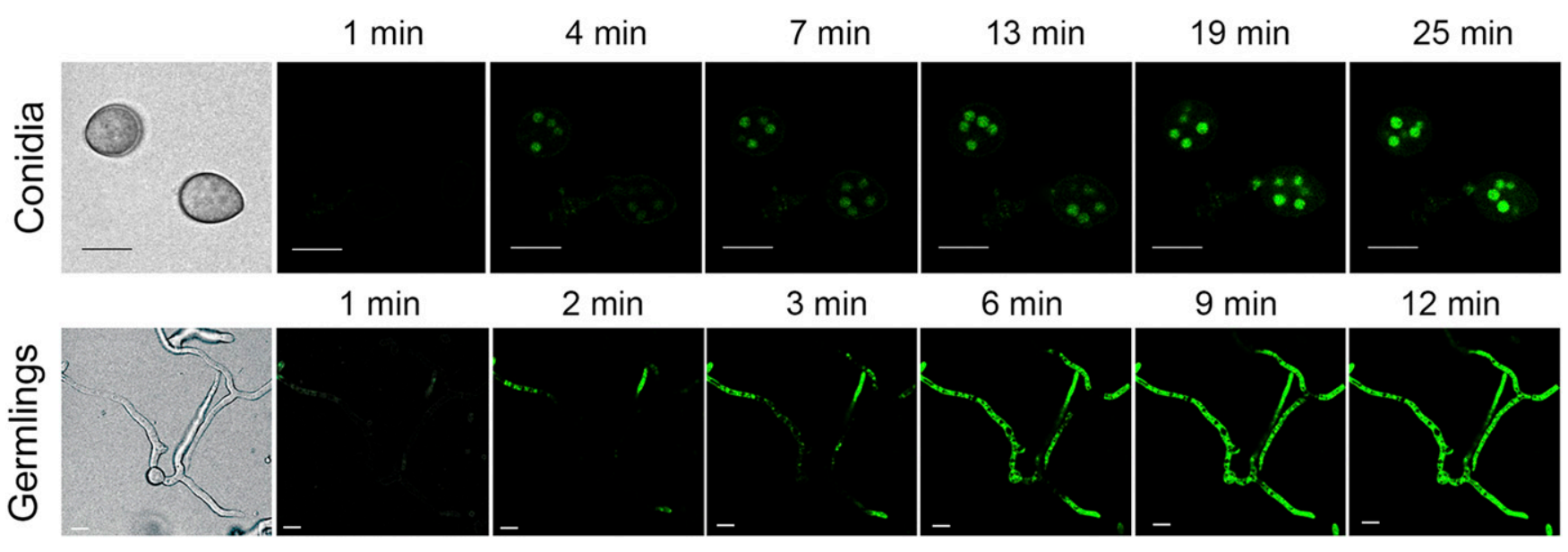

Fig. 3. OefDef1.1 induces membrane permeabilization both in conidia and germlings of Botrytis cinerea. B. cinerea conidia and germlings were incubated with $3 \mu \mathrm{M}$ OefDef1.1 and $1 \mu \mathrm{M}$ SYTOX Green. Confocal microscopy images were taken every 3 min for conidia and every 1 min for germlings after OefDef1.1 challenge. Bars $=10 \mu \mathrm{m}$.
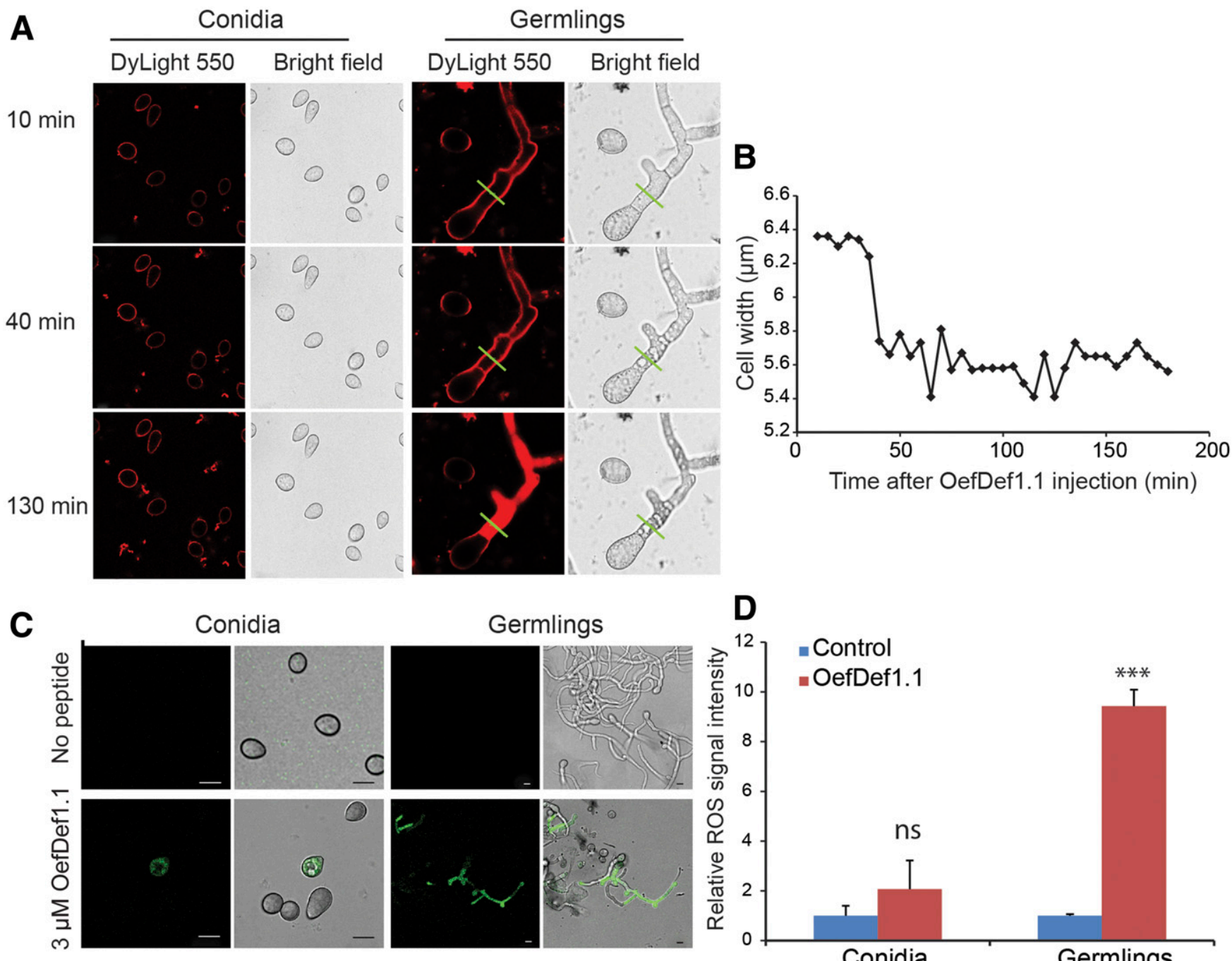

Fig. 4. The conidia and germlings of Botrytis cinerea differ in their uptake of OefDef1.1 and reactive oxygen species (ROS) production. A, The intracellular localization of $6 \mu \mathrm{M}$ DyLight 550-labeled OefDef1.1 in B. cinerea conidia and germlings. The confocal microscope images were captured every 5 min after OefDef1.1 challenge. Images taken at 10, 40, and $130 \mathrm{~min}$ are shown. B, Cell width was determined by quantifying the inner distance between cell walls in bright field images from the germling cell shown in A (the measuring location is labeled by green lines in A). C, Confocal microscope images showing ROS production (green fluorescence) in fresh conidia and germlings after treatment with $3 \mu \mathrm{M}$ OefDef1.1 for 90 min. Bars $=10 \mu \mathrm{m}$. D, The relative ROS signal intensity in fresh conidia and germlings. Samples without OefDef1.1 treatment were used as controls. The statistical analysis was performed by two-tailed unpaired $t$ test to determine whether the observed differences were statistically significant $(P<0.001)$ as shown by asterisks. ns $=$ not significant $(P>0.05)$. Error bars denote standard deviation values. 
effective in controlling gray mold disease than peptides with a lower net charge. These results indicate that the in vitro antifungal activity of the peptide often does not correlate with the in planta antifungal activity.

\section{Antifungal activity of OefDef1.1_V1 is more tolerant} to mono- and divalent cations than that of OefDef1.1.

Antifungal activity of some plant defensins is significantly reduced in growth media containing elevated mono- and divalent cations, potentially limiting their efficacy as antifungal agents in vivo (Osborn et al. 1995; Spelbrink et al. 2004). It has been hypothesized that the presence of cations significantly weakens the electrostatic interactions between a positively charged defensin and negatively charged fungal membranes (Melo et al. 2009). We therefore evaluated antifungal activity of wild-type OefDef1.1 and its more positively charged OefDef1.1_V1 variant in a low-salt synthetic fungal medium (SFM) (Liang et al. 2001) supplemented with $100 \mathrm{mM} \mathrm{NaCl}$, $100 \mathrm{mM} \mathrm{KCl}$, or $1 \mathrm{mM} \mathrm{CaCl}_{2}$. As expected, antifungal activity of the wild-type OefDef1.1 is significantly reduced in the elevated concentration of each cation in the SFM. Even at a $4 \times$ MIC concentration of $6 \mu \mathrm{M}$, significant conidial germination was observed in the presence of each cation (Fig. 7A). Surprisingly, OefDef1.1_V1 at 0.75 and $1.5 \mu \mathrm{M}$ exhibited more potent antifungal activity in the presence of $100 \mathrm{mM} \mathrm{NaCl}$ or $100 \mathrm{mM} \mathrm{KCl}$ than in its absence (Fig. 7A). OefDef1.1_V1 exhibits similar antifungal activity in vitro, with or without the addition of $1 \mathrm{mM} \mathrm{CaCl}{ }_{2}$. These results were further confirmed by a resazurin cell-viability assay (Supplementary Fig. S9). The cationic media used in this study slightly inhibited the growth of $B$. cinerea, but most conidia and mycelium were still metabolically alive. These results indicate that increased cationicity positively correlates with the higher antifungal activity of the peptide in the presence of cations.

A high-quality homology-based model was also generated for OefDef1.1_V1. A comparison of the solvent-accessible surface potential plots of the 3D structures of OefDef1.1 and OefDef1.1_V1 indicated the presence of a highly charged pocket similar to that recently reported for maize defensin
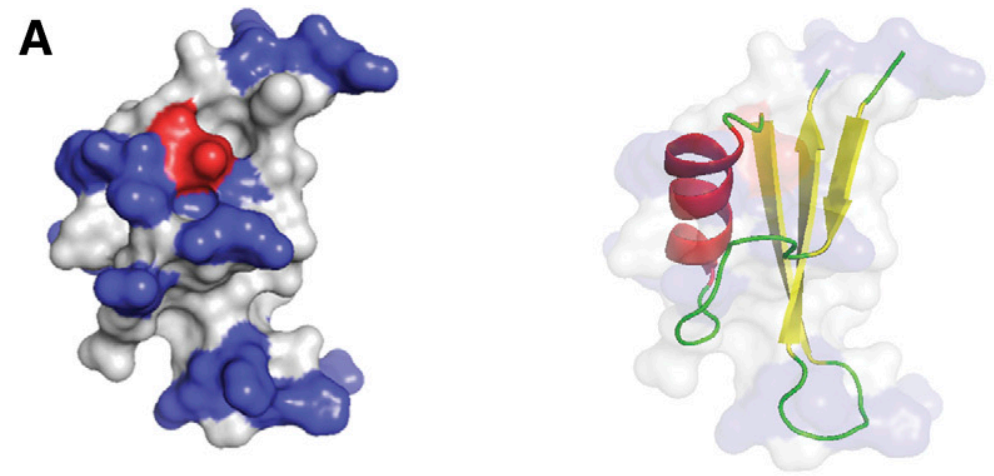

B

OefDef1.1 KPCTKLSKGWRGLCAPHKCSSYCIHHEGAYHGACLKNRHSKHYGCYCYYRHCY OefDef1.1_V1 KPCTKLSKGWRGLCAPHKCSSYCIHHEGAYHGRCRGFRRR CYCYYRHCY OefDef1.1_V2 KPCTKLSKGWRGLCAPHKCSSYCIHHEGAYHGACHVRNGKHM CYCYYRHCY
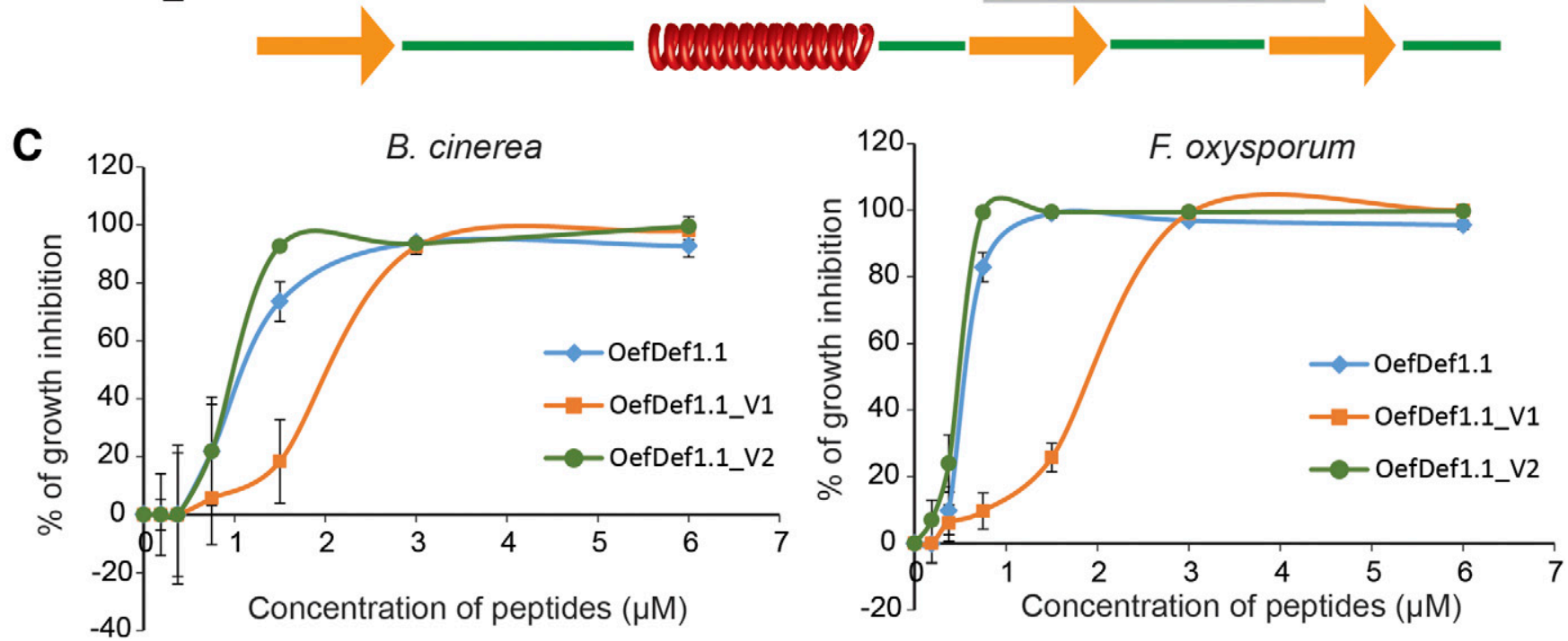

Fig. 5. Homology-based three-dimensional (3D) structure of OefDef1.1 and antifungal activity of its $\gamma$-core motif variants. A, 3D structure and the surface of the wild-type OefDef1.1 were constructed by homology-based modeling. The surface representation of OefDef1.1 (left) displays the cationic residues in blue, anionic residues in red, and uncharged or hydrophobic residues in white. The cartoon representation (right) displays $\alpha$-helix in red, $\beta$-sheets in yellow, and loops in green. The Artemisia vulgaris Artv1 (PDB number 2KPY) was used as the template. B, The amino acid sequences of the wild-type OefDef1.1 and its variants. The $\gamma$-core motifs of OefDef1.1 and its two variants are shown in the yellow rectangle, and $\gamma$-core motif sequences selected for substitution are shown in red. The $\alpha$-helix is shown as a red spiral, three $\beta$-sheets are shown as orange arrows, and the loops are shown as green lines. $\mathbf{C}$, Antifungal activity of the wildtype OefDef1.1 and its variants against Botrytis cinerea and F. oxysporum. Error bars denote standard deviation values. 

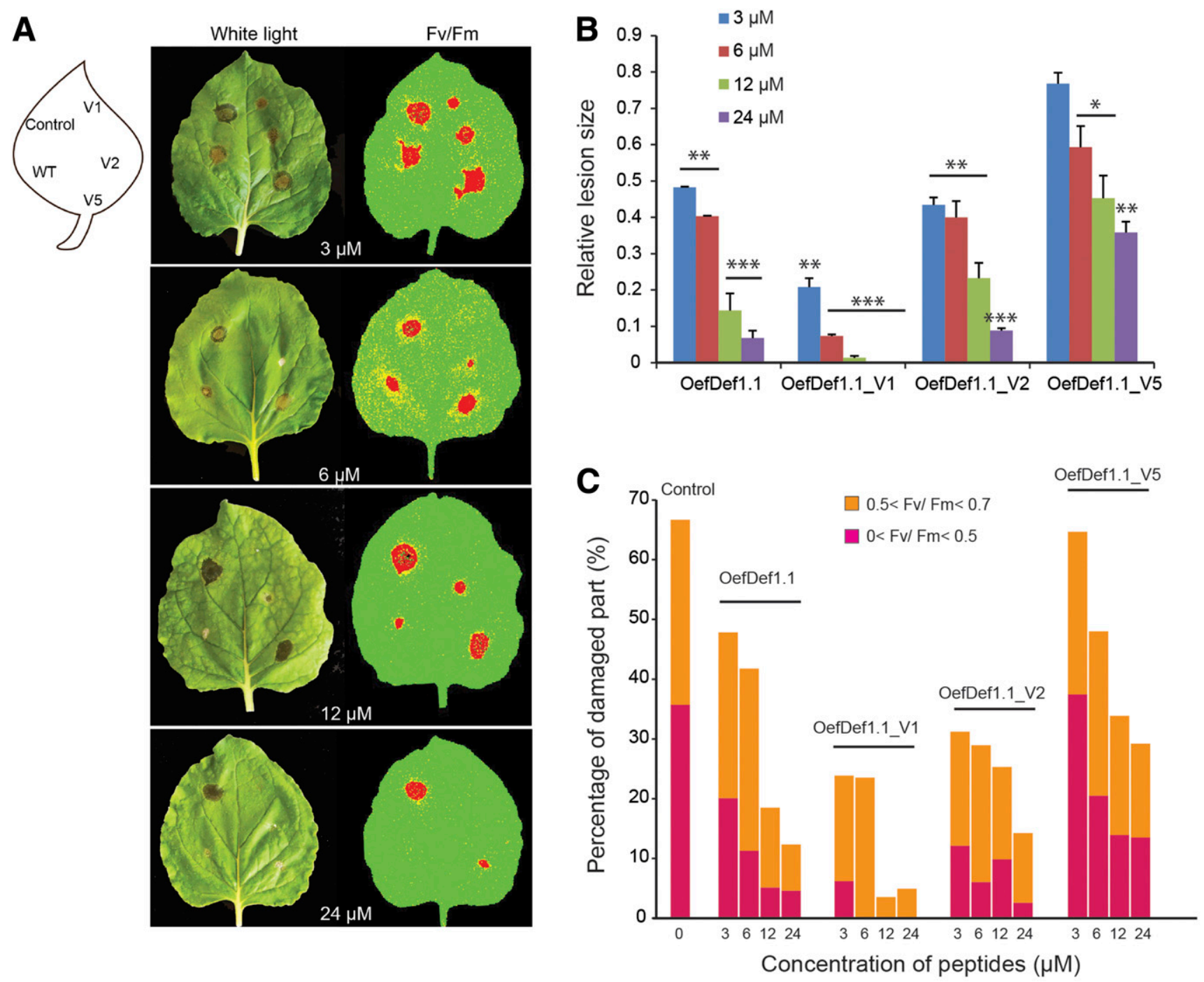

D

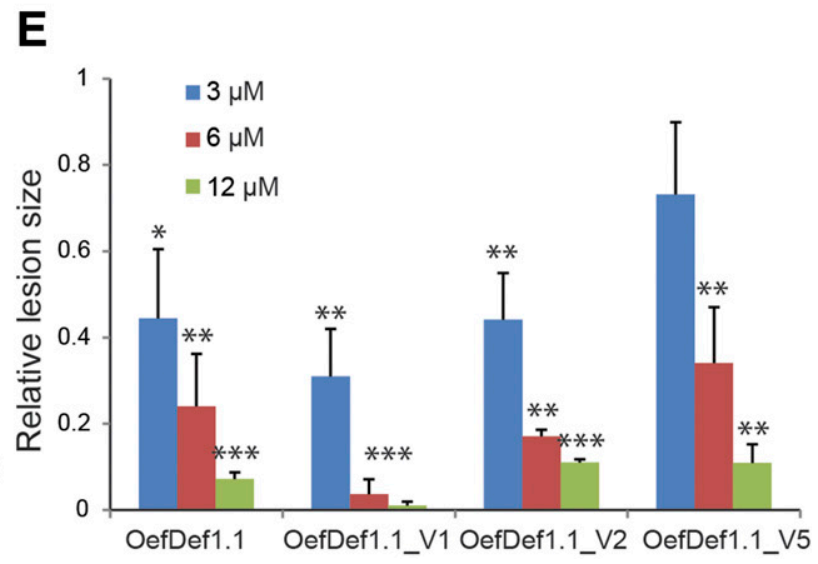

Fig. 6. The inhibition of gray mold disease symptoms on the Nicotiana benthamiana and lettuce leaves by topical application of OefDef 1.1 and variants. A, The lesions formed by Botrytis cinerea were imaged under white light and high-resolution images were taken by CropReporter based on the value of potential photosynthetic efficiency ( $\mathrm{Fv} / \mathrm{Fm}$ ). The value of $\mathrm{Fv} / \mathrm{Fm}$ was shown as different colors (red, Fv/Fm $<0.5$; yellow, $0.5<\mathrm{Fv} / \mathrm{Fm}<0.7$; green, $\mathrm{Fv} / \mathrm{Fm}>0.7$ ). Different concentrations $(0,3,6,12$, and $24 \mu \mathrm{M})$ of OefDef1.1, OefDef1.1_V1, OefDef1.1_V2, and OefDef1.1_V5 were applied onto the leaves first and fresh conidia of $B$. cinerea were applied at the same spot. Images were captured after $48 \mathrm{~h}$ postinoculation (hpi). B, The relative lesion size was measured from the white light images, using the ImageJ software. The lesion formed without peptide was used as a control. C, The percentage of damaged part of each $35 \times 35$ pixel frame containing the whole lesion of each treatment. Fv/Fm values for the red and orange columns are indicated. The lesions caused without peptide treatment were set as control (100\%). D, Lesions formed by B. cinerea on lettuce leaves applied with 3, 6, and 12 $\mu$ M of OefDef1.1, OefDef1.1_V1, OefDef1.1_V2, and OefDef1.1_V5. The control samples are highlighted by a red rectangle. E, The relative lesion sizes on lettuce leaves as determined by ImageJ software. The control sample with no peptide applied was set as 1 . Statistical analysis was performed by two-tailed unpaired $t$ test to determine whether the observed differences were statistically significant (one asterisk [*], $P<0.05$; two [**], $P<0.01$; three [***], $P<0.001$ ). Error bars denote standard deviation value. Three independent replications were performed for each experiment. 
ZmD32 (Kerenga et al. 2019) in OefDef1.1_V1 but not in OefDef1.1 (Fig. 7B). We hypothesize that the formation of a cationic pocket on the protein surface might be responsible for the enhanced antifungal activity of OefDef1.1_V1 in the presence of elevated $\mathrm{Na}^{1+}, \mathrm{K}^{1+}$, and $\mathrm{Ca}^{2+}$.
OefDef1.1_V1 exhibits stronger binding affinity to multiple membrane phospholipids than does OefDef1.1.

To gain further insight into the MOA of OefDef1.1 and OefDef1.1_V1, we assessed their interaction with various bioactive phospholipids by performing a protein-lipid overlay

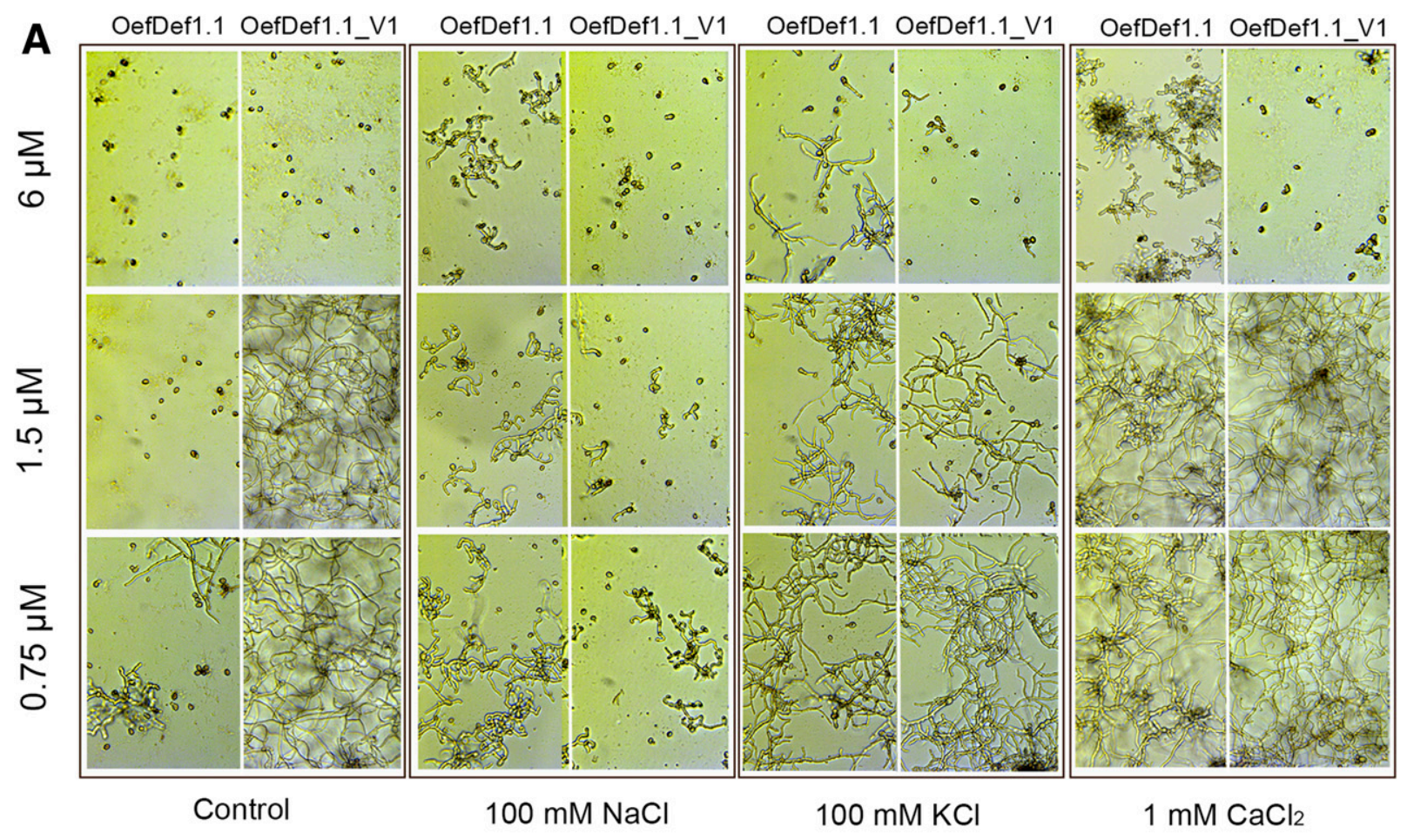

B
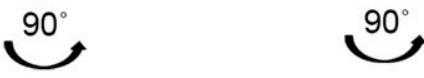

$90^{\circ}$

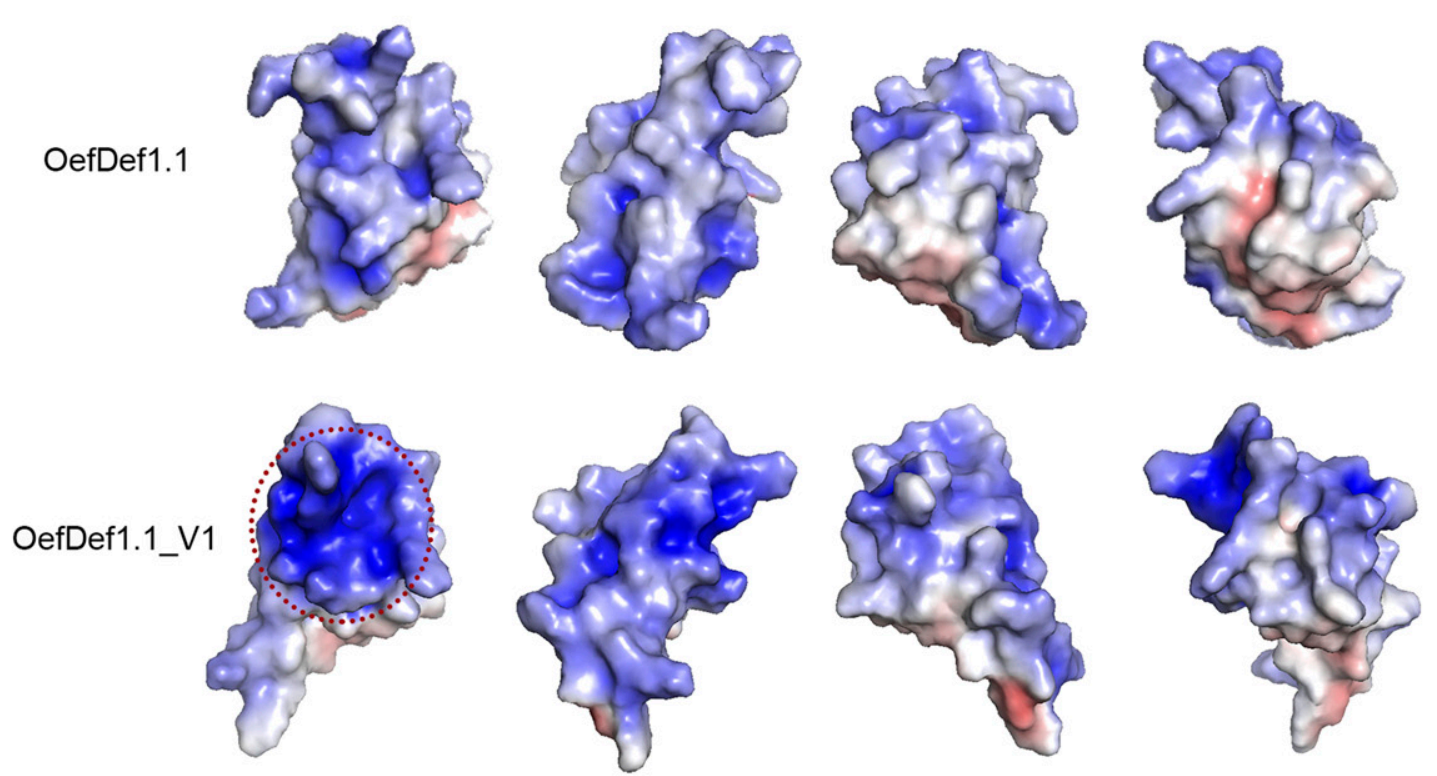

$-5.000$

Fig. 7. Antifungal activity of OefDef1.1 and OefDef1.1_V1 against Botrytis cinerea in the presence of mono- and bivalent cations and surface charge of these peptides. A, The growth of $B$. cinerea treated with OefDef1.1 and OefDef1.1_V1 in media containing cations. Images were captured by microscopy at $60 \mathrm{~h}$ postinoculation (hpi). B, Qualitative electrostatic surface representation of OefDef1.1 and OefDef1.1_V1 (cationic residues are represented in blue, anionic residues in red, and uncharged or hydrophobic residues in white). The cationic pocket is highlighted by a red dotted circle. The surface charges are based on the dielectric constant at \pm 5 (bar). 
assay. Commercially available PIP (phosphoinositide) strips containing a 100-pmole spot for each of the 15 phospholipids were used for this assay. First, the binding affinity of the primary anti-OeDef1.1 polyclonal antibody to each peptide was examined (Supplementary Fig. S10). The polyclonal antibody showed much weaker binding affinity to OefDef1.1_V1 than to the wild-type OefDef1.1. OefDef1.1 antibody produced a strong signal upon binding to $1 \mu \mathrm{g}$ of each peptide. It also produced strong signal upon binding to $500 \mathrm{ng}$ of OefDef1.1 but not to OefDef1.1_V1. Second, when this antibody was used in the lipid-protein overlay assay, OefDef1.1 showed binding to phosphatidylinositol 3-phosphate (PI3P) and PI5P and relatively weak binding to PI4P, PI3, $5 \mathrm{P}_{2}$, and phosphatidic acid (PA). Surprisingly, OefDef1.1_V1 showed stronger binding to the same phospholipids than OefDef1.1, despite the fact that the antibody recognized the variant poorly. We conclude that the replacement of its own $\gamma$-core motif with that of MtDef4 significantly enhances the phospholipid binding ability of OefDef1.1.

\section{Antifungal properties of OefDef1.1 vary significantly among closely related ascomycete fungal pathogens.}

In order to determine if the antifungal mechanisms of OefDef1.1 are conserved in closely related ascomycete fungal pathogens $F$. graminearum, $F$. virguliforme, $F$. oxysporum, and $B$. cinerea, we examined the ability of OefDef1.1 to permeabilize the plasma membrane and enter into the interior of the conidia and germlings of each pathogen in SFM or SFM containing $100 \mathrm{mM} \mathrm{NaCl}$. In addition, we determined the antifungal activity of OefDef1.1 against each pathogen, as determined by $100 \%$ inhibition of conidial germination in the presence of $100 \mathrm{mM} \mathrm{NaCl}$. In SFM without elevated $\mathrm{Na}^{1+}$, OefDef1.1 permeabilized the plasma membrane of conidia in all pathogens. As expected, additional $\mathrm{Na}^{1+}$ significantly reduced the ability of OefDef1.1 to permeabilize the plasma membrane of conidia in each pathogen, but quantitative differences were observed among pathogens (Table 2; Supplementary Fig. S11). In low-salt medium, more than $90 \%$ of the $B$. cinerea and $F$. virguliforme conidia were permeabilized by OefDef1.1. However, in the presence of elevated $\mathrm{Na}^{1+}$, only $3.9 \%$ of $B$. cinerea conidia were permeabilized while $35.8 \%$ of $F$. virguliforme conidia were permeabilized by this defensin.

Significant quantitative differences were also noted in the internalization of OefDef1.1 into conidia of these pathogens in SFM without elevated $\mathrm{Na}^{1+}$ (Table 2). For example, the conidia of the three Fusarium spp. but not of $B$. cinerea internalized OefDef1.1. In the presence of elevated $\mathrm{Na}^{1+}$, uptake of OefDef1.1 was inhibited in all four pathogens, although $24.3 \%$ of $F$. virguliforme conidia still retained the ability to internalize the peptide. In contrast, internalization of the peptide was completely blocked in the conidia of $F$. oxysporum in the presence of high $\mathrm{Na}^{1+}$. Thus, the conidia of the two Fusarium spp. responded differently to the antifungal action of OefDef 1.1 in the presence of a high concentration of the monovalent cation. Consistent with these results, antifungal activity of OefDef1.1 was retained against $F$. virguliforme in the presence of $\mathrm{Na}^{1+}$ but not against the other three fungi (Table 2; Supplementary Fig. S12).

The ability of OefDef1.1 to permeabilize the plasma membrane and enter into cytoplasm of germlings was almost completely blocked in the presence of high $\mathrm{Na}^{1+}$ in $B$. cinerea, $F$. graminearum, and $F$. oxysporum. However, in $F$. virguliforme germlings, the uptake of SG and DyLight 550-labeled peptide was still clearly visible (Fig. 8). Based on these results, we conclude that the antifungal properties of OefDef1.1 can vary even in closely related fungal pathogens.

\section{DISCUSSION}

In this study, we identified a novel gene family encoding highly cationic histidine- and tyrosine-rich defensins in the evergreen perennial woody plants olive and European ash trees. This family of defensins appears to be unique to the family Oleaceae. Since the annotation of the olive tree and the European ash tree draft genomes is still far from complete, the exact number of these genes in the genome of each tree remains to be determined with certainty. Nine defensins in the olive tree share more than $90 \%$ sequence identity and, thus, appear to have evolved as a result of recent gene duplication. The uniqueness of these defensins also suggests that they have likely evolved to perform specific biological functions in the Oleaceae family. They are among the most positively charged defensin peptides in the plant kingdom and contain a high percentage of hydrophobic residues. However, they each contain a cationic $\gamma$-core motif different in sequence and length from those in other wellcharacterized defensins.

In the present study, we have found that OefDef1.1 exhibits potent broad-spectrum antifungal activity against four closely related ascomycete fungal pathogens and it significantly reduces the symptoms of gray mold disease caused by $B$. cinerea infection semi-in planta. The ability of OefDef1.1 to inhibit fungal pathogen growth in vitro and semi-in planta leads us to predict that this defensin plays a role in host defense against fungal pathogens in olive tree.

Antifungal plant defensins vary in their MOA. However, they all share a common characteristic of interacting with the fungal cell wall and permeabilizing the plasma membrane of fungal pathogens (Cools et al. 2017; Parisi et al. 2019). Very little is known, at this time, regarding the cell-wall components interacting with plant defensins. In our mechanistic studies employing $B$. cinerea as our model fungus, we determined that OefDef1.1 quickly binds to the cell walls of conidia and germlings. However, in the presence of $100 \mathrm{mM} \mathrm{NaCl}$, it still binds to the cell wall of conidia but very weakly to that of germlings, indicating its cell type-specific interaction with the cell wall.

Fungal cell wall is a dynamic organelle, and its structure and composition display significant variations between different cell types and growth conditions within the same species (Geoghegan et al. 2017). Thus, the relative concentrations of $\beta 1,3$-glucan, $\beta 1,6$-glucan, chitin, mannan, and glycoproteins, the major components of the cell wall, might vary between cell

Table 2. Percentage of plasma membrane permeabilization and defensin internalization in conidia from different fungal pathogens ${ }^{\mathrm{a}}$

\begin{tabular}{|c|c|c|c|c|c|}
\hline \multirow[b]{2}{*}{ Pathogens } & \multicolumn{2}{|c|}{ Permeabilization (\%) } & \multicolumn{2}{|c|}{ Internalization (\%) } & \multirow[b]{2}{*}{$\operatorname{MIC}(\boldsymbol{\mu M})$} \\
\hline & $-\mathbf{N a C l}$ & $+\mathrm{NaCl}$ & $-\mathrm{NaCl}$ & $+\mathrm{NaCl}$ & \\
\hline B. cinerea & $93.5 \pm 1.7$ & $3.9 \pm 1.7$ & $4.2 \pm 1.6$ & $2.5 \pm 1.8$ & $>6$ \\
\hline F. graminearum & $65.3 \pm 3.1$ & $15.2 \pm 3.1$ & $33.2 \pm 4.9$ & $7.8 \pm 1.8$ & $>6$ \\
\hline F. virguliforme & $98.0 \pm 1.8$ & $35.8 \pm 7.1$ & $93.6 \pm 3.0$ & $24.3 \pm 5.1$ & 1.5 \\
\hline F. oxysporum & $51.3 \pm 5.5$ & $7.1 \pm 2.1$ & $46.6 \pm 11.0$ & $0.9 \pm 0.3$ & $>6$ \\
\hline
\end{tabular}

${ }^{a}$ Values are mean \pm standard deviation; $n=3$. MIC value is based on resazurin cell-viability assay in the presence of $100 \mathrm{mM}$ NaCl. 
types. Significant changes in the major polysaccharides of the cell wall occur when a $B$. cinerea conidium germinates and differentiates into a germling (Cantu et al. 2009; Ruiz-Herrera 1992). Significant changes in the carbohydrate composition of the cell wall at different stages (spore, mycelium, and zygote) of development have also been reported in Penicillium roqueforti and Absidia coerulea (Andriyanova et al. 2011). Fungal cell wall also comprises a complex matrix of interconnected polysaccharides and proteins (Latgé 2007). Thus, differences in the cell-wall proteomes of the conidia and germlings could account for cell type-specific differences observed in the cell-wall binding of OefDef1.1 in the presence of a monovalent cation $\mathrm{Na}^{1+}$.

Our data revealed notable differences among three Fusarium spp. and B. cinerea with regard to the ability of OefDef1.1 to

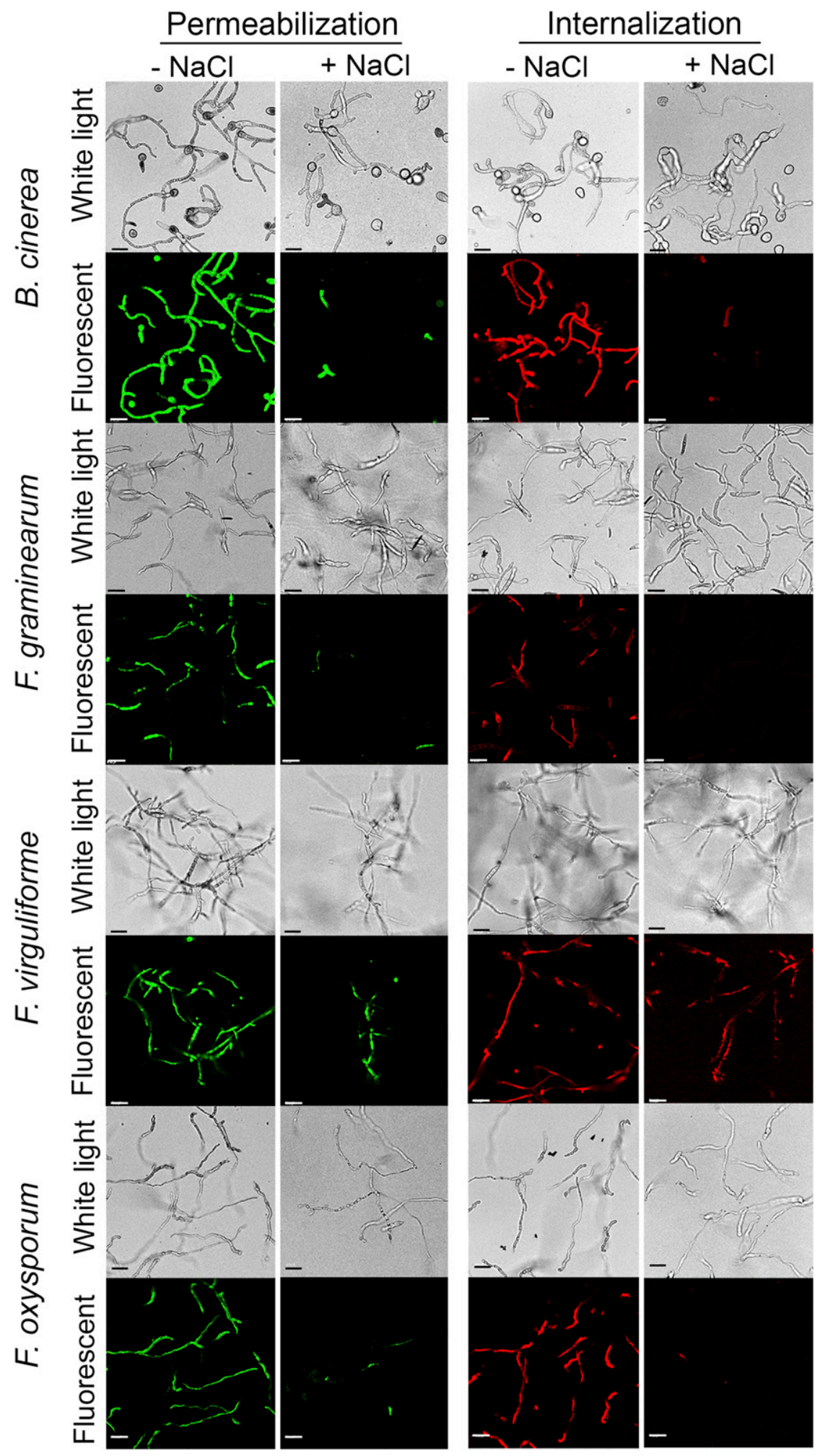

Fig. 8. Plasma membrane permeabilization of fungal cells and internalization of OefDef1.1 into fungal cells in different pathogens incubated with or without $100 \mathrm{mM} \mathrm{NaCl}$. Germlings of each fungal pathogen were treated with $2 \times$ minimum inhibitory concentration values of peptide. Images were captured after $3 \mathrm{~h}$ of defensin challenge. SYTOX Green was used at $1 \mu \mathrm{M}$. Bars $=20 \mu \mathrm{m}$. 
permeabilize the plasma membrane, gain entry into conidial and germling cells, and kill fungal cells in the absence and presence of elevated $\mathrm{Na}^{1+}$. It is particularly interesting that, in the presence of high $\mathrm{Na}^{1+}$, OefDef1.1 permeabilizes the plasma membrane and enters the conidial and germling cells of $F$. virguliforme but not of closely related $F$. oxysporum. These observations are noteworthy, especially since the two Fusarium spp. belong to the same Nectriaceae family and exhibit hemibiotrophic lifestyles. Although molecular targets of OefDef1.1 in the cell wall of fungal pathogens used in this study are not yet known, significant differences must exist in the composition and architecture of the cell wall of closely related fungal species of the phylum Ascomycota to account for varied responses of the fungal species to OefDef1.1 (Geoghegan et al. 2017).

OefDef1.1 rapidly permeabilizes the plasma membrane of $B$. cinerea, suggesting that it is a critical event in the fungicidal action of this defensin. Membrane permeabilization has been shown to be an important early step in the MOA of several plant defensins such as RsAFP2, DmAMP1, HsAFP1, MsDef1, MtDef4, and NaD1 (Cools et al. 2017; Parisi et al. 2019). Some of these defensins bind to sphingolipids, while others engage PA or phoshoinositides or both and induce membrane permeabilization (Cools et al. 2017; Parisi et al. 2019). The protein-lipid overlay assay used in this study revealed binding of OefDef1.1 to multiple phospholipids including PIPs and PA. Surprisingly, the OefDef1.1 variant containing the $\gamma$-core motif of MtDef4 exhibits stronger binding to the same phospholipids. However, more quantitative assays such as the surface plasmon resonance and liposome binding assays are needed to determine the relative binding affinity of each peptide to one or more if its phospholipid partners. To what extent the enhanced phospholipid binding of OefDef1.1_V1 contributes to its greater semiin planta antifungal activity against $B$. cinerea remains an open question.

Induction of ROS is hypothesized to be another important event in the antifungal action of plant defensins, including NaD1 (van der Weerden et al. 2008), RsAFP2 (Aerts et al. 2007), and MtDef5 (Islam et al. 2017b). OefDef1.1 challenge also induces ROS production in germlings, suggesting their involvement in cell death. However, the ROS signal intensity induced by OefDef1.1 in freshly harvested conidia only accounts for $20 \%$ of the ROS signal in germlings. These data suggest that ROS are likely not involved in the killing of conidia by OefDef1.1. Whether they contribute to the killing of germlings by this defensin remains to be determined.

Several plant defensins have now been shown to be translocated inside the fungal cells (Cools et al. 2017; Islam et al. 2017b; Parisi et al. 2019). It has been proposed that these defensins bind to intracellular targets as part of their MOA. Internalization of OefDef 1.1 appears to be cell type-dependent and varies among fungal species. In $B$. cinerea, OefDef1.1 remains bound to the cell wall of conidia and almost no uptake (less than 5\%) is observed, even after $3 \mathrm{~h}$ of challenge. However, it is internalized rapidly in the germlings of this pathogen. In contrast, this defensin is internalized in both cell types of the fungal pathogens, $F$. virguliforme and $F$. oxysporum. To what extent internalization of this peptide contributes to the killing of fungal cells in these pathogens remains to be determined. We propose that internalization is not required for killing of the conidial cells in $B$. cinerea.

The structure of OefDef1.1 is similar to those of other plant defensins. However, its $\gamma$-core motif (GACLKNRHSKHYGC) is different from those of other well-characterized plant defensins in length and sequence. The $\gamma$-core motif contains major determinants for phospholipid binding, oligomer formation, and antifungal activity of several plant defensins (Cools et al. 2017; De Coninck et al. 2013; Parisi et al. 2019; Sagaram et al. 2012). The analysis of the in vitro and semi-in planta antifungal activity of two chimeric OefDef1.1 variants, each containing a heterologous $\gamma$-core motif, yielded unexpected results. OefDef1.1_V1 containing the $\gamma$-core motif sequence (GRCRGFRRRC) of MtDef4 was less active against $B$. cinerea than the wild-type OefDef1.1 in vitro, but it provided greater control of this pathogen semi-in planta. In contrast, OefDef1.1_V2 containing the $\gamma$-core motif sequence (CHVRNGKHMC) of DmAMP1 was more active than the wild-type OefDef1.1 in vitro but provided similar control of this pathogen semi-in planta. Thus, under the assay conditions used, in vitro antifungal activity of a plant defensin does not necessarily correlate with semi-in planta antifungal activity. One likely explanation for this apparent discrepancy is that OefDef1.1_V1 exhibits greater antifungal activity in the presence of elevated $\mathrm{Na}^{1+}$ and $\mathrm{K}^{1+}$ than does OefDef1.1. It is likely that the antifungal activity of OefDef1.1_V1, to a large extent, is independent of the exogenous monovalent cations. Addition of exogenous $\mathrm{Ca}^{2+}$ is known to curtail the antifungal activity of plant defensins (Osborn et al. 1995; Spelbrink et al. 2004). Thus, it is not surprising that the addition of $1 \mathrm{mM} \mathrm{Ca}^{2+}$ abrogates the antifungal activity of OefDef1.1. It is, however, surprising that exogenous $\mathrm{Ca}^{2+}$ at $1 \mathrm{mM}$ has no effect on the antifungal activity of OefDef1.1_V1 at all concentrations tested. This may be because $\mathrm{Ca}^{2+}$ is unable to compete with this peptide for binding to the negatively charged fungal cell wall or the perturbation of calcium homeostasis caused by exogenous $\mathrm{Ca}^{2+}$ is not enough to affect the OeDef1.1_V1-fungal pathogen interaction. The ability to retain antifungal activity in the presence of monovalent and bivalent cations may be important for a plant defensin to confer resistance to a fungal pathogen semi-in planta. Recently, highly cationic maize defensin ZmD32 retaining broad-spectrum antifungal activity in media containing elevated levels of $\mathrm{Na}^{1+}$ has been characterized (Kerenga et al. 2019). As in OefDef1.1_V1, ZmD32 also contains a positively charged pocket in its 3D structure. It is proposed that the RGFRRR motif present in the $\gamma$-core motif of each defensin is responsible for the formation of this cationic pocket and cation-tolerant antifungal activity.

Based on the MOA studies reported here, we propose a multistep model for the antifungal action of OefDef 1.1 against B. cinerea (Fig. 9). Although no difference was observed in the MIC value of this defensin between conidia and germlings (data not shown), their interactions with this peptide were significantly different. In both conidia and germlings, the first two steps are initial binding to the cell wall followed by rapid disruption of the plasma membrane. $\mathrm{Na}^{1+}$ blocks cell-wall binding of the peptide in germlings. While, in conidia, $\mathrm{Na}^{1+}$ does not block binding of the peptide to the cell wall, it does block membrane permeabilization. After $90 \mathrm{~min}$ of peptide challenge, significantly more ROS is detected in germlings than in conidia. Within $3 \mathrm{~h}$ of peptide challenge, massive internalization of the peptide and significant cell shrinkage is observed in germlings but not in conidia. Better understanding of the relative contribution of each step to the antifungal action of this defensin will require further studies. However, rapid membrane permeabilization stands out as a major contributing factor to the antifungal mechanism of OefDef1.1.

\section{MATERIALS AND METHODS}

Fungal cultures and growth medium.

The fungal strains Fusarium graminearum $\mathrm{PH}-1$, F. virguliforme NRRL 22292 (Mont-1), F. oxysporum f. sp. cubense, and Botrytis cinerea T-4 were cultured in media shown in Supplementary Table $\mathrm{S} 2$. F graminearum and $F$. virguliforme were gifts of J.-R. Xu at Purdue University and A. Fakhoury at Southern Illinois University, 
respectively. The $F$. oxysporum isolate was from R. Beachy at the Donald Danforth Plant Science Center and B. cinerea was a gift of M. Dickman of Texas A \& M University.

Defensin gene identification and phylogenetic analysis.

Defensin genes were identified through BLASTP, using the default parameters on Phytozome, a plant genomics resource portal, and National Center for Biotechnology Information databases using as queries peptide sequences of MtDef4 and MtDef5 (Islam et al. 2017b; Sagaram et al. 2011). All other defensin sequences were obtained from the available published literature. The mature peptide sequences were aligned using multiple sequence alignment tool MUSCLE, and the phylogenetic tree was inferred by the neighbor-joining method, using the Geneious software with bootstrap $(10,000$ replications).

\section{Recombinant expression and purification of OefDef1.1 and its variants in Pichia pastoris.}

The codon-optimized OefDef1.1, OefDef1.1_V1, and OefDef1.1_V2 genes were synthesized by GenScript. The genes of variants OefDef1.1_V3, OefDef1.1_V4, and OefDef1.1_V5 were generated using the QuickChange II site-directed mutagenesis kit (Agilent Technologies). The target DNA fragments were inserted into pPICZ $\alpha$ vector and were introduced into Pichia pastoris X33 by electroporation. Zeocin resistance was used as a selectable marker for identification of transformants. The selected transformant expressing each peptide was cultured in BMGY $(1.34 \%$ YNB, $1 \%$ yeast extract, $2 \%$ peptone, $10 \mathrm{mM}$ potassium phosphate buffer, $\mathrm{pH}$ 6.0, $0.4 \mathrm{~g}$ of biotin per liter, and $1 \%$ glycerol) medium for $24 \mathrm{~h}$. Cells were harvested by centrifugation at $5,000 \times g$ for $15 \mathrm{~min}$ and were resuspended into BMMY (BMGY but with $0.5 \%$ methanol instead of $1 \%$

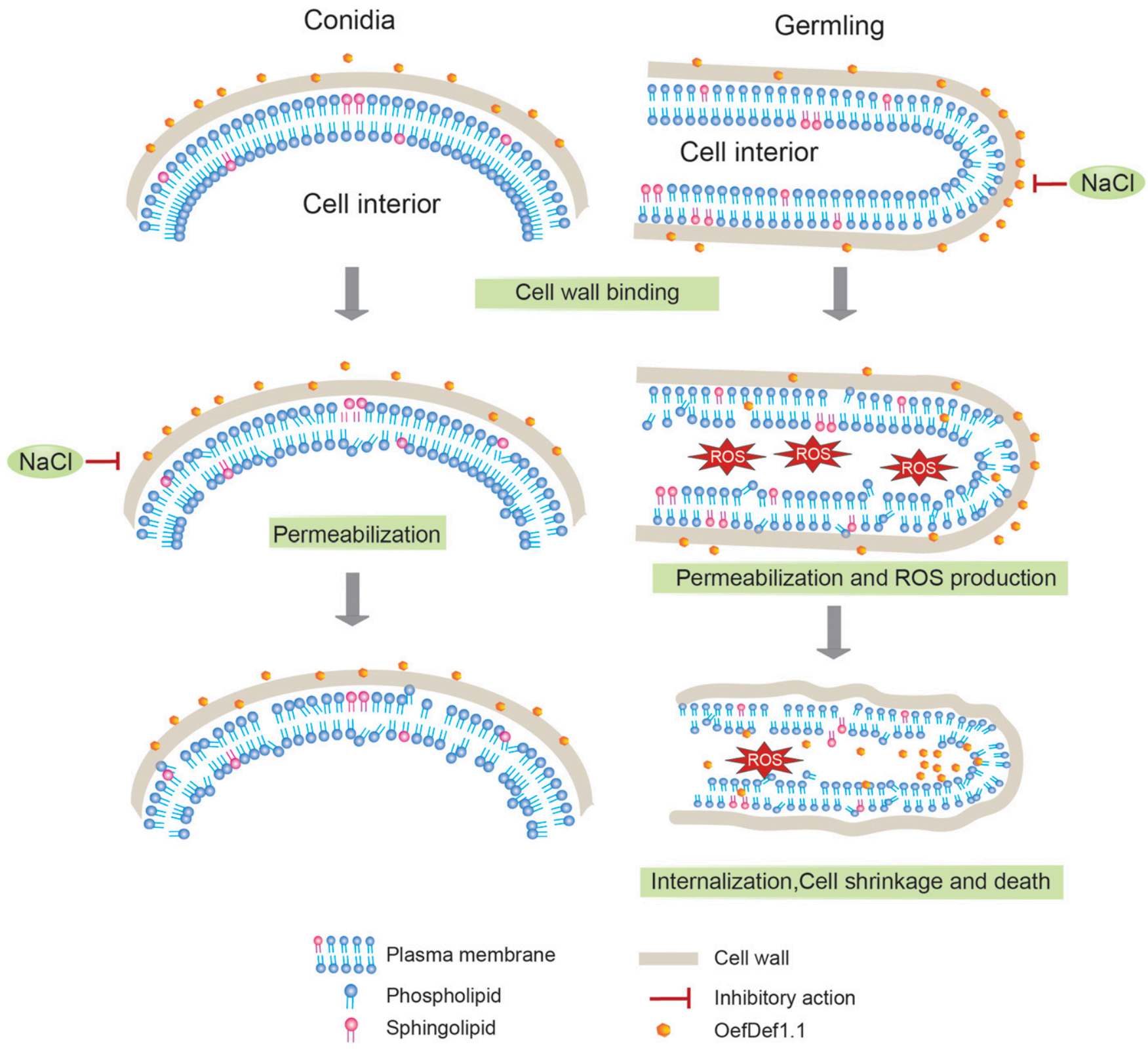

Fig. 9. Proposed models showing differences in the fungicidal action of OefDef1.1 against the conidia and germlings of $B$. cinerea. OefDef1.1 binds to the cell wall and permeabilizes the plasma membrane of conidia and germlings. However, $\mathrm{NaCl}$ blocks cell-wall binding of the peptide in germlings but not in conidia, and also blocks membrane permeabilization in conidia. After $90 \mathrm{~min}$ of peptide challenge, significantly more reactive oxygen species are detected in germlings than in conidia. Within $3 \mathrm{~h}$ of peptide challenge, massive internalization of the peptide and significant cell shrinkage is observed in germlings, but not in conidia. 
glycerol) media. Methanol was added every $24 \mathrm{~h}$ to $0.5 \%$ (vol/vol) final concentration for inducing target peptide expression. After 5 days, OefDef 1.1 and its variants were each purified from the culture medium, using the Fast Protein liquid chromatography system (GE Healthcare) with CM Sephadex C-25 (Sigma-Aldrich), reverse phase-high performance liquid chromatography with C18 column (Alltech), and, subsequently, were lyophilized as described (Islam et al. 2017a and b). Each peptide was resuspended in nuclease-free water and its concentration was determined by either NanoDrop spectrophotometry or the BCA assay. Purity and size of each peptide were determined by electrophoresis on 4 to $20 \%$ Mini-Protean TGX gels (Bio-Rad). The correct mass of each peptide was confirmed by mass spectrometry, using a Triversa Nanomate nanospray direct infusion robot (Advion) attached to a LTQ OrbiTrap Velos Pro Mass Spectrometer (Thermo-Fisher Scientific) prior to its use in experiments described below.

\section{In vitro antifungal activity assays.}

Antifungal activity assays were conducted as described previously with minor modifications (Spelbrink et al. 2004). The quantitative fungal growth inhibition by OefDef1.1 and its variants was estimated by measuring the absorbance at $595 \mathrm{~nm}$ using a Tecan Infinite M200 Pro (Tecan Systems Inc.) microplate reader at $48 \mathrm{~h}$. The fungal cell killing was determined by the resazurin cell-viability assay (Chadha and Kale 2015). After incubation of the pathogen-peptide mixture for $48 \mathrm{~h}, 10 \mu \mathrm{l}$ of $0.1 \%$ resazurin solution was added to each well and was reincubated overnight. A change in the color of the resazurin dye from blue to pink or colorless indicated the presence of live fungal cells.

Effect of cations on the antifungal activity of OefDef1.1 and OefDef1_V1 against $B$. cinerea conidia was tested, as described above, in the presence of $100 \mathrm{mM} \mathrm{NaCl}, 100 \mathrm{mM} \mathrm{KCl}$, or $1 \mathrm{mM} \mathrm{CaCl}_{2}$. The plates were incubated at room temperature for $60 \mathrm{~h}$, and images were taken on a Leica DMI 6000B microscope.

\section{Semi-in planta antifungal activity of OefDef1.1 and its variants.}

Detached leaf infection assays were performed as described (Wang et al. 2016), with minor modifications. Briefly, leaves of iceberg lettuce and $N$. benthamiana were placed in petri dishes. A 10- $\mu$ l aliquot containing different concentrations of each defensin was placed onto the leaf samples, and inoculation with $B$. cinerea was initiated at the same spot by applying $10 \mu \mathrm{l}$ of spore suspension at a final concentration of $10^{5}$ spores per milliliter of SFM buffer. Petri dishes were kept in Ziploc WeatherShield plastic boxes containing wet paper towels at room temperature for $48 \mathrm{~h}$. Lesions were photographed and the relative lesion sizes were determined using ImageJ software. The biomass of fungal pathogen was determined by qPCR with primers shown in Supplementary Table S3. Briefly, the same size plant materials containing the whole lesions were collected from different treatments. The samples were ground in liquid nitrogen and $100 \mathrm{mg}$ of the homogenized sample was used for genomic DNA extraction, using the fungal DNA mini kit (Omega Bio-tek). A pair of primers specific for B. cinerea internal transcribed spacer gene and lettuce actin gene were used to amplify 192 and $98 \mathrm{bp}$ fragments, respectively. The $10-\mu \mathrm{l}$ reaction system included $5 \mu \mathrm{l}$ of $2 \times$ SsoAdvanced universal probes supermix (Bio-Rad), $1 \mu \mathrm{l}$ of diluted cDNA, $0.2 \mu \mathrm{l}$ of each primer (10 pmol per microliter), and $3.6 \mu$ of doubledistilled $\mathrm{H}_{2} \mathrm{O}$. The quantitative reverse transcription-PCR was performed in quadruplicate on the Bio-Rad (CFX384) machine and using the following program: initial denaturation of $2 \mathrm{~min}$ at $95^{\circ} \mathrm{C}$, followed by 40 amplification cycles of $5 \mathrm{~s}$ at $95^{\circ} \mathrm{C}, 30 \mathrm{~s}$ at $60^{\circ} \mathrm{C}, 30 \mathrm{~s}$ at $72^{\circ} \mathrm{C}$. The leaf tissue inoculated with $B$. cinerea but no peptide was used as control. The ratio of $B$. cinerea to lettuce genomic DNA was calculated using the $-\Delta \Delta$ cycle threshhold method.

The high-resolution images of the $B$. cinerea infection of $N$. benthamiana leaves were obtained using CropReporter (PhenoVation). The chlorophyll fluorescence and Fv/Fm (ratio of variable fluorescence to saturation level of fluorescence) images of the infected leaves were captured using the fluorescence imaging technology (Gorbe et al. 2015).

\section{Plasma membrane permeabilization.}

Membrane permeabilization of fungal cells was analyzed using confocal microscopy by visualizing the influx of the fluorescent dye SG (Thermo Fisher Scientific). Fresh conidia or germlings mixed with $2 \times \mathrm{MIC}$ ( $3 \mu \mathrm{M}$ for $B$. cinerea and $F$. oxysporum and $6 \mu \mathrm{M}$ for $F$. graminearum and $F$. virguliforme) OefDef1.1 and $1 \mu \mathrm{M}$ SG were deposited onto glass-bottom petri dishes and were imaged by confocal microscopy at an excitation wavelength of $488 \mathrm{~nm}$ and an emission wavelength ranging from 520 to $600 \mathrm{~nm}$ at specific time intervals. Plates with SG but without OefDef1.1 were used as negative controls. A Leica SP8-X confocal microscope was used for all confocal imaging, and Leica LASX (version 3.1.5.) software was used to process these images. The percentage of conidia showing the membrane permeabilization was determined by counting the number of cells showing fluorescent signal relative to the total number of cells counted.

\section{Uptake of OefDef1.1 by fungal cells.}

OefDef1.1 was labeled with DyLight 550 amine reactive dye following the protocol provided by the manufacturer (Thermo Fisher Scientific). Time-lapse confocal laser scanning microscopy was performed, to monitor uptake and subcellular localization of the fluorescently labeled peptide, as described previously (Islam et al. 2017a). Since labeled OefDef1.1 lost approximately $50 \%$ of its antifungal activity, it was used at a final concentration of $6 \mu \mathrm{M}$ for $B$. cinerea and $F$. oxysporum or $12 \mu \mathrm{M}$ for $F$. graminearum and $F$. virguliforme. For colocalization assay, DyLight 550-labeled OefDef1.1 was added to $B$. cinerea conidia along with $5 \mu \mathrm{M}$ membrane-selective dye FM4-64. The excitation and emission wavelength for DyLight 550 were 562 and 580 to $680 \mathrm{~nm}$ and, for FM4-64, 690 and $800 \mathrm{~nm}$, respectively. The percentage of conidia showing internalization was determined by counting the number of conidial cells showing fluorescent signal relative to the total number of conidial cells counted.

\section{Intracellular ROS detection.}

Intracellular ROS were detected in fresh conidia and 16-hold germlings after exposure to $3 \mu \mathrm{M}$ OefDef1.1 for $90 \mathrm{~min}$. After treatment, $2^{\prime}, 7^{\prime}$-dichlorodihydrofluorescein diacetate (Sigma-Aldrich) was added at a final concentration of $10 \mu \mathrm{M}$, and live-cell imaging was performed using the Leica SP8-X microscope, as described previously (Islam et al. 2017a).

\section{Structure prediction.}

The predicted 3D structures of OefDef1.1 and OefDef1.1_V1 were generated using the SWISS-MODEL online server and software PyMOL 0.99rc6 (DeLano Scientific LLC). The 3D structure of the Artemisia vulgaris Artv1 defensin (PDB 2KPY) was used as a template. The structures generated were refined by the GalaxyWEB server. The quality of the predicted model was evaluated using ERRAT and Procheck online tools.

\section{Phospholipid-protein overlay assay.}

Phospholipid-protein overlay assay was performed as described Islam et al. (2017b). Briefly, the assay employed PIP 
strips, which are spotted with 100 pmol of various biologically active phospholipids (Echelon Biosciences). The binding of OefDef1.1 and OefDef1.1_V1 to their phospholipid partners was detected using the affinity-purified OefDef1.1-derived peptide (HHEGAYHGACLKNRHSKHYGCYCYYRHCY) polyclonal antibody (GenScript) and horseradish peroxidase-conjugated goat anti-rabbit immunoglobulin G secondary antibody (Sigma-Aldrich), following manufacturer protocol. The signals were detected using the SuperSignal West Femto maximum sensitivity substrate (Thermo Fisher Scientific). To determine the binding affinity of peptides to primary antibody, different amounts of OefDef1.1 and OefDef1.1_V1 (1 $\mu \mathrm{g}, 500 \mathrm{ng}$, and $100 \mathrm{ng})$ were spotted onto nitrocellulose membrane $(0.2-\mu \mathrm{m}$ pore size $)$ and were incubated at room temperature for $30 \mathrm{~min}$ with $1 \mu \mathrm{g}$ of primary antibody per millililiter. The nitrocellulose membrane was processed in the same manner as the PIP strips described above.

\section{ACKNOWLEDGMENTS}

We thank S. C. Tzeng and B. Evans of the Proteomics and Mass Spectrometry Facility for conducting mass spectrometry analysis of OefDef1.1 and its variants. We thank H. Berg of the Integrated Microscopy Facility for his expert guidance and help with confocal microscopy.

\section{AUTHOR-RECOMMENDED INTERNET RESOURCES}

Defensins Sequence Space database: https://ts404.shinyapps.io/defspace ERRAT tool: http://servicesn.mbi.ucla.edu/ERRAT GalaxyWEB server: http://galaxy.seoklab.org

Procheck tool: http://www.ebi.ac.uk/thornton-srv/databases/pdbsum/Generate.html The SWISS-MODEL online server: www.swissmodel.expasy.org

\section{LITERATURE CITED}

Aerts, A. M., François, I. E., Meert, E. M., Li, Q. T., Cammue, B. P., and Thevissen, K. 2007. The antifungal activity of RsAFP2, a plant defensin from raphanus sativus, involves the induction of reactive oxygen species in Candida albicans. J. Mol. Microbiol. Biotechnol. 13:243-247.

Andriyanova, D. A., Sergeeva, Y. E., Kochkina, G. A., Galanina, L. A., Usov, A. I., and Feofilova, E. P. 2011. Filamentous fungi's cell-wall extraction at different stages of ontogenesis and exploration of their carbohydrate composition. Appl. Biochem. Microbiol. 47:405-411.

Barghini, E., Natali, L., Cossu, R. M., Giordani, T., Pindo, M., Cattonaro, F., Scalabrin, S., Velasco, R., Morgante, M., and Cavallini, A. 2014. The peculiar landscape of repetitive sequences in the olive (Olea europaea L.) genome. Genome Biol. Evol. 6:776-791.

Cantu, D., Greve, L. C., Labavitch, J. M., and Powell, A. L. 2009. Characterization of the cell wall of the ubiquitous plant pathogen Botrytis cinerea. Mycol. Res. 113:1396-1403.

Chadha, S., and Kale, S. P. 2015. Simple fluorescence-based high throughput cell viability assay for filamentous fungi. Lett. Appl. Microbiol. 61:238-244.

Cools, T. L., Struyfs, C., Cammue, B. P., and Thevissen, K. 2017. Antifungal plant defensins: Increased insight in their mode of action as a basis for their use to combat fungal infections. Future Microbiol. 12: 441-454.

Cruz, F., Julca, I., Gómez-Garrido, J., Loska, D., Marcet-Houben, M., Cano, E., Galán, B., Frias, L., Ribeca, P., Derdak, S., Gut, M., SánchezFernández, M., García, J. L., Gut, I. G., Vargas, P., Alioto, T. S., and Gabaldón, T. 2016. Genome sequence of the olive tree, Olea europaea. Gigascience 5:29.

De Coninck, B. M., Cammue, B. P. A., and Thevissen, K. 2013. Modes of antifungal action and in planta functions of plant defensins and defensinlike peptides. Fungal Biol. Rev. 26:109-120.

Dean, R., Van Kan, J. A., Pretorius, Z. A., Hammond-Kosack, K. E., Di Pietro, A., Spanu, P. D., Rudd, J. J., Dickman, M., Kahmann, R., Ellis, J., and Foster, G. D. 2012. The top 10 fungal pathogens in molecular plant pathology. Mol. Plant Pathol. 13:414-430.

El-Mounadi, K., Islam, K. T., Hernández-Ortiz, P., Read, N. D., and Shah, D. M. 2016. Antifungal mechanisms of a plant defensin MtDef4 are not conserved between the ascomycete fungi Neurospora crassa and Fusarium graminearum. Mol. Microbiol. 100:542-559.

Geoghegan, I., Steinberg, G., and Gurr, S. 2017. The role of the fungal cell wall in the infection of plants. Trends Microbiol. 25:957-967.
Giacomelli, L., Nanni, V., Lenzi, L., Zhuang, J., Dalla Serra, M., Banfield, M. J., Town, C. D., Silverstein, K. A., Baraldi, E., and Moser, C. 2012. Identification and characterization of the defensin-like gene family of grapevine. Mol. Plant-Microbe Interact. 25:1118-1131.

Gorbe, E., Heuvelink, E., Jalink, H., and Stanghellini, C. 2015. Effect of low temperature during the night in young sweet pepper plants: Stress and recovery. Acta Hortic.: 115-121.

Goyal, R. K., and Mattoo, A. K. 2014. Multitasking antimicrobial peptides in plant development and host defense against biotic/abiotic stress. Plant Sci. 228:135-149.

Islam, K. T., Shah, D. M., and El-Mounadi, K. 2017a. Live-cell imaging of fungal cells to investigate modes of entry and subcellular localization of antifungal plant defensins. J. Vis. Exp. 130:e55995.

Islam, K. T., Velivelli, S. L. S., Berg, R. H., Oakley, B., and Shah, D. M. 2017b. A novel bi-domain plant defensin MtDef5 with potent broadspectrum antifungal activity binds to multiple phospholipids and forms oligomers. Sci. Rep. 7:16157.

Kaur, J., Sagaram, U. S., and Shah, D. M. 2011. Can plant defensins be used to engineer durable commercially useful fungal resistance in crop plants? Fungal Biol. Rev. 25:128-135.

Kerenga, B. K., McKenna, J. A., Harvey, P. J., Quimbar, P., Garcia-Ceron, D., Lay, F. T., Phan, T. K., Veneer, P. K., Vasa, S., Parisi, K., Shafee, T. M. A., van der Weerden, N. L., Hulett, M. D., Craik, D. J., Anderson, M. A., and Bleackley, M. R. 2019. Salt-tolerant antifungal and antibacterial activities of the corn defensin ZmD32. Front. Microbiol. 10:795.

Lacerda, A. F., Vasconcelos, E. A., Pelegrini, P. B., and Grossi de Sa, M. F. 2014. Antifungal defensins and their role in plant defense. Front Microbiol. 5:116.

Latgé, J. P. 2007. The cell wall: A carbohydrate armour for the fungal cell Mol. Microbiol. 66:279-290.

Liang, J., Shah, D. M., Wu, Y., Rosenberger, C. A., and Hakimi, S. M. 2001. Antifungal polypeptide from alfalfa and methods for controlling plant pathogenic fungi. U.S. patent 6,316,407.

Melo, M. N., Ferre, R., and Castanho, M. A. 2009. Antimicrobial peptides: Linking partition, activity and high membrane-bound concentrations. Nat. Rev. Microbiol. 7:245-250.

Nanni, V., Schumacher, J., Giacomelli, L., Brazzale, D., Sbolci, L., Moser, C., Tudzynski, P., and Baraldi, E. 2014. VvAMP2, a grapevine flowerspecific defensin capable of inhibiting Botrytis cinerea growth: Insights into its mode of action. Plant Pathol. 63:899-910.

Osborn, R. W., De Samblanx, G. W., Thevissen, K., Goderis, I., Torrekens, S., Van Leuven, F., Attenborough, S., Rees, S. B., and Broekaert, W. F. 1995. Isolation and characterisation of plant defensins from seeds of Asteraceae, Fabaceae, Hippocastanaceae and Saxifragaceae. FEBS Lett. 368:257-262.

Parisi, K., Shafee, T. M. A., Quimbar, P., van der Weerden, N. L., Bleackley, M. R., and Anderson, M. A. 2019. The evolution, function and mechanisms of action for plant defensins. Semin. Cell Dev. Biol. 88: 107-118.

Poon, I. Kh., Baxter, A. A., Lay, F. T., Mills, G. D., Adda, C. G., Payne, J. A., Phan, T. K., Ryan, G. F., White, J. A., Veneer, P. K., van der Weerden, N. L., Anderson, M. A., Kvansakul, M., and Hulett, M. D. 2014. Phosphoinositide-mediated oligomerization of a defensin induces cell lysis. eLife 3:e01808.

Ruiz-Herrera, J. 1992. Fungal cell wall structure, synthesis and assembly. CRC Press, Boca Raton, FL, U.S.A.

Sagaram, U., Kaur, J., and Shah, D. 2012. Antifungal plant defensins: structure-activity relationships and modes of action and biotech applications. Pages 317-336 in: Small wonders: peptides for disease control. K. Rajasekaran, ed. American Chemical Society, Washington, D.C.

Sagaram, U. S., Pandurangi, R., Kaur, J., Smith, T. J., and Shah, D. M. 2011 Structure-activity determinants in antifungal plant defensins MsDef1 and MtDef4 with different modes of action against Fusarium graminearum. PLoS One 6:e18550.

Shafee, T. M., Lay, F. T., Hulett, M. D., and Anderson, M. A. 2016. The defensins consist of two independent, convergent protein superfamilies. Mol. Biol. Evol. 33:2345-2356.

Sollars, E. S., Harper, A. L., Kelly, L. J., Sambles, C. M., Ramirez-Gonzalez, R. H., Swarbreck, D., Kaithakottil, G., Cooper, E. D., Uauy, C., Havlickova, L., Worswick, G., Studholme, D. J., Zohren, J., Salmon, D. L., Clavijo, B. J., Li, Y., He, Z., Fellgett, A., McKinney, L. V., Nielsen, L. R., Douglas, G. C., Kjær, E. D., Downie, J. A., Boshier, D., Lee, S., Clark, J., Grant, M., Bancroft, I., Caccamo, M., and Buggs, R. J. 2017. Genome sequence and genetic diversity of European ash trees. Nature 541:212-216.

Spelbrink, R. G., Dilmac, N., Allen, A., Smith, T. J., Shah, D. M., and Hockerman, G. H. 2004. Differential antifungal and calcium channel-blocking 
activity among structurally related plant defensins. Plant Physiol. 135: 2055-2067.

Thevissen, K., de Mello Tavares, P., Xu, D., Blankenship, J., Vandenbosch, D., Idkowiak-Baldys, J., Govaert, G., Bink, A., Rozental, S., de Groot, P. W., Davis, T. R., Kumamoto, C. A., Vargas, G., Nimrichter, L., Coenye, T., Mitchell, A., Roemer, T., Hannun, Y. A., and Cammue, B. P. 2012. The plant defensin RsAFP2 induces cell wall stress, septin mislocalization and accumulation of ceramides in Candida albicans. Mol. Microbiol. 84:166-180.

Thevissen, K., François, I. E. J. A., Takemoto, J. Y., Ferket, K. K. A., Meert, E. M. K., and Cammue, B. P. A. 2003. DmAMP1, an antifungal plant defensin from dahlia (Dahlia merckii), interacts with sphingolipids from Saccharomyces cerevisiae. FEMS Microbiol. Lett. 226:169-173.

Unver, T., Wu, Z., Sterck, L., Turktas, M., Lohaus, R., Li, Z., Yang, M., He, L., Deng, T., Escalante, F. J., Llorens, C., Roig, F. J., Parmaksiz, I., Dundar, E., Xie, F., Zhang, B., Ipek, A., Uranbey, S., Erayman, M., Ilhan,
E., Badad, O., Ghazal, H., Lightfoot, D. A., Kasarla, P., Colantonio, V., Tombuloglu, H., Hernandez, P., Mete, N., Cetin, O., Van Montagu, M., Yang, H., Gao, Q., Dorado, G., and Van de Peer, Y. 2017. Genome of wild olive and the evolution of oil biosynthesis. Proc. Natl. Acad. Sci. U.S.A. 114:E9413-E9422.

van der Weerden, N. L., and Anderson, M. A. 2013. Plant defensins: Common fold, multiple functions. Fungal Biol. Rev. 26:121-131.

van der Weerden, N. L., Bleackley, M. R., and Anderson, M. A. 2013. Properties and mechanisms of action of naturally occurring antifungal peptides. Cell. Mol. Life Sci. 70:3545-3570.

van der Weerden, N. L., Lay, F. T., and Anderson, M. A. 2008. The plant defensin, NaD1, enters the cytoplasm of Fusarium oxysporum hyphae. J. Biol. Chem. 283:14445-14452.

Wang, M., Weiberg, A., Lin, F. M., Thomma, B. P. H. J., Huang, H. D., and Jin, H. 2016. Bidirectional cross-kingdom RNAi and fungal uptake of external RNAs confer plant protection. Nat. Plants 2:16151. 